\title{
Dimension-six matrix elements for meson mixing and lifetimes from sum rules
}

\section{Kirk, A. Lenz and T. Rauh}

IPPP, Department of Physics, University of Durham, DH1 3LE, United Kingdom

E-mail: m.j.kirk@durham.ac.uk, alexander.lenz@durham.ac.uk,

thomas.j.rauh@durham.ac.uk

ABSTRACT: The hadronic matrix elements of dimension-six $\Delta F=0,2$ operators are crucial inputs for the theory predictions of mixing observables and lifetime ratios in the $B$ and $D$ system. We determine them using HQET sum rules for three-point correlators. The results of the required three-loop computation of the correlators and the one-loop computation of the QCD-HQET matching are given in analytic form. For mixing matrix elements we find very good agreement with recent lattice results and comparable theoretical uncertainties. For lifetime matrix elements we present the first ever determination in the $D$ meson sector and the first determination of $\Delta B=0$ matrix elements with uncertainties under control - superseeding preliminary lattice studies stemming from 2001 and earlier. With our stateof-the-art determination of the bag parameters we predict: $\tau\left(B^{+}\right) / \tau\left(B_{d}^{0}\right)=1.082_{-0.026}^{+0.022}$, $\tau\left(B_{s}^{0}\right) / \tau\left(B_{d}^{0}\right)=0.9994 \pm 0.0025, \tau\left(D^{+}\right) / \tau\left(D^{0}\right)=2.7_{-0.8}^{+0.7}$ and the mixing-observables in the $B_{s}$ and $B_{d}$ system, in good agreement with the most recent experimental averages.

KEYwords: Effective Field Theories, Heavy Quark Physics, Nonperturbative Effects

ArXIV EPRINT: 1711.02100 


\section{Contents}

1 Introduction 1

2 QCD-HQET matching for $\Delta B=2$ operators 3

2.1 Setup 3

2.2 Results 5

2.3 Matching of QCD and HQET Bag parameters 6

$\begin{array}{lll}3 & \text { HQET sum rule } & 7\end{array}$

$\begin{array}{ll}3.1 & \text { The sum rule }\end{array}$

3.2 Spectral functions at NLO 8

$\begin{array}{lll}3.3 & \text { Sum rule for the Bag parameters } & 11\end{array}$

4 Results for $\Delta B=2$ operators $\quad 12$

$\begin{array}{lll}4.1 & \text { Details of the analysis } & 13\end{array}$

$\begin{array}{lll}4.2 & \text { Results and comparison } & 14\end{array}$

$\begin{array}{lll}4.3 & B_{s} \text { and } B_{d} \text { mixing observables } & 16\end{array}$

$5 \Delta B=0$ operators and ratios of $B$-meson lifetimes $\quad 17$

$\begin{array}{lll}5.1 & \text { Operators and matrix elements } & 17\end{array}$

$\begin{array}{lll}5.2 & \text { Results for the spectral functions and bag parameters } & 18\end{array}$

$\begin{array}{lll}5.3 & \text { Results for the lifetime ratios } & 21\end{array}$

6 Matrix elements for charm and the $D^{+}-D^{0}$ lifetime ratio 21

6.1 Matrix elements for $D$ mixing 21

6.2 Matrix elements for $D$ lifetimes and $\tau\left(D^{+}\right) / \tau\left(D^{0}\right) \quad 23$

$\begin{array}{lll}7 & \text { Conclusions } & 24\end{array}$

A Basis of evanescent operators and ADMs $\quad 26$

A.1 $\Delta B=2$ operators $\quad 26$

A.2 $\Delta B=0$ operators 28

$\begin{array}{ll}\text { B Inputs and detailed overview of uncertainties } & 29\end{array}$

\section{Introduction}

The mixing of neutral mesons proceeds through flavour-changing neutral currents and is therefore loop suppressed in the Standard Model. Thus, mixing observables are very sensitive to new physics effects. Our ability to constrain new contributions strongly relies on a high degree of precision in both experiment and theory. Mixing is most pronounced in the $B_{s}$ system where the relative decay rate difference amounts to about $13 \%$. Here the experimental precision has surpassed the theoretical one by a significant margin [1]. 
The theory expression for mixing observables is a product of perturbative coefficients and non-perturbative matrix elements. The perturbative part is known up to NLO-QCD (see the discussion below) and first steps in the direction of a NNLO-QCD evaluation have recently been performed by [2]. However, the dominant theoretical uncertainties still stem from hadronic matrix elements of local $\Delta B=2$ four-quark operators. They are usually determined by lattice simulations and results for the leading dimension-six operators are available from several collaborations [3-5]. If only the latest lattice results [5] are used, small tensions at the level of two sigma emerge in $B_{s}$ mixing $[5,6]$. To either settle or solidify this issue, an independent determination of the matrix elements and further scrutinization of the theoretical methods are necessary. We address both these points in this paper.

An alternative way to determine hadronic matrix elements is given by QCD sum rules $[7,8]$. This approach employs quark-hadron duality and the analyticity of Green functions instead of the discretization of space-time. Thus, its sources of uncertainties are entirely different from lattice simulations and sum rule analyses can provide truly independent results. We determine the hadronic matrix elements of the dimension-six $\Delta B=2$ operators for $B$-mixing from a sum rule for three-point correlators first introduced in [9]. The sum rule is valid at scales $\mu_{\rho} \sim 1.5 \mathrm{GeV}$ which are much smaller than the bottomquark mass. Therefore the sum rule is formulated in HQET, where quantum fluctuations with a characteristic scale of the order of the bottom-quark mass have been integrated out. We then run the HQET matrix elements up to a scale $\mu_{m}$ of the order of the bottom-quark mass where the matching to QCD can be performed without introducing large logarithms. Earlier sum rule results are available for the SM operator $Q_{1}[10,11]$ and condensate corrections have been computed for dimension-six [11-15] and seven [12, 13] operators. The same strategy is then applied to determine the matrix elements of dimension-six $\Delta B=0$ operators, which are the non-perturbative input for calculating ratios of lifetimes of different mesons, like $\tau\left(B^{+}\right) / \tau\left(B_{d}\right)$ and $\tau\left(B_{s}\right) / \tau\left(B_{d}\right)$, see e.g. [16] for a review. Here the perturbative part of the prediction is also known to NLO-QCD.

The theory prediction for the $B_{s}$ decay rate difference $\Delta \Gamma_{s}$ and for ratios of lifetimes of different $B$ mesons is based on the Heavy Quark Expansion (HQE) [17-20]. The HQE is an OPE in the Minkowski domain which has fuelled speculations about large violations of duality, in particular for $\Delta \Gamma_{s}$ which is dominated by the $b \rightarrow c \bar{c} s$ transition. ${ }^{1}$ A recent confrontation of $\mathrm{HQE}$ predictions with experiment has ruled out duality violations larger than about $20 \%$ [6]. Ratios of meson lifetimes are a good testing ground for the validity of the HQE, but have suffered from large hadronic uncertainties [16] in the past because only outdated lattice results $[22,23]$ for the required $\Delta B=0$ matrix elements of four quark operators were available. We present the first state-of-the-art calculation of the $\Delta B=0$ matrix elements and determine the lifetimes with significantly reduced uncertainties.

In the charm sector the validity of the HQE is rather uncertain due to its smaller mass $m_{c} \sim m_{b} / 3$. The direct translation of the predictions for $B$ mixing fails by several orders of magnitude [24]. However it has been argued that higher-dimensional contributions can lift

\footnotetext{
${ }^{1}$ Interestingly we find that the HQE prediction for the $b \rightarrow c \bar{c} s$ branching ratio [21] is in excellent agreement with experiment.
} 
the severe GIM suppression in the charm sector and potentially explain the size of mixing observables [24-28]. D-meson lifetimes have been studied recently [29] and have shown no indications for a breakdown of the HQE, albeit with large hadronic uncertainties. We translate our sum rule results to the charm sector as well. The $\Delta C=2$ matrix elements show good agreement with lattice results [30-32] and the $\Delta C=0$ results are used to update the $D^{+}-D^{0}$ lifetime ratio.

The outline of this work is as follows: in section 2 we describe the details of the QCD-HQET matching computation focussing on $\Delta B=2$ operators. The sum rule and the calculation of the three-point correlators are discussed in section 3. Our results for the matrix elements are presented in section 4 and compared to other recent works. In section 5 we study $\Delta B=0$ operators and ratios of $B$-meson lifetimes. We determine the matrix elements of $\Delta C=0,2$ operators in section 6 and update the HQE result for the $D^{+}-D^{0}$ lifetime ratio using these results. Finally, we conclude in section 7 .

\section{QCD-HQET matching for $\Delta B=2$ operators}

We perform the matching computation between QCD and HQET operators at the one-loop level. The details of the computation are described in section 2.1 for the $\Delta B=2$ operators. Our results for the matching of the operators and Bag parameters are given in section 2.2 and section 2.3 , respectively.

\subsection{Setup}

The matching calculation for the SM operator $Q_{1}$ appearing in $\Delta M_{s}$ has been performed in [33-35]. We compute the matching coefficients of the full dimension-six $\Delta B=2$ operator basis needed for $\Delta M_{s}$ in BSM theories and for $\Delta \Gamma_{s}$ in the SM. We work in dimensional regularization with $d=4-2 \epsilon$ and an anticommuting $\gamma^{5}$ (NDR scheme). We consider the following operators in QCD

$$
\begin{array}{ll}
Q_{1}=\bar{b}_{i} \gamma_{\mu}\left(1-\gamma^{5}\right) q_{i} \bar{b}_{j} \gamma^{\mu}\left(1-\gamma^{5}\right) q_{j}, & \\
Q_{2}=\bar{b}_{i}\left(1-\gamma^{5}\right) q_{i} \bar{b}_{j}\left(1-\gamma^{5}\right) q_{j}, & Q_{3}=\bar{b}_{i}\left(1-\gamma^{5}\right) q_{j} \bar{b}_{j}\left(1-\gamma^{5}\right) q_{i}, \\
Q_{4}=\bar{b}_{i}\left(1-\gamma^{5}\right) q_{i} \bar{b}_{j}\left(1+\gamma^{5}\right) q_{j}, & Q_{5}=\bar{b}_{i}\left(1-\gamma^{5}\right) q_{j} \bar{b}_{j}\left(1+\gamma^{5}\right) q_{i} .
\end{array}
$$

To fix the renormalization scheme we also have to specify a basis of evanescent operators [36-38]. We do this following [39]. The explicit form of the evanescent operators can be found in appendix A. On the HQET side, we have the operators

$$
\begin{array}{ll}
\tilde{Q}_{1}=\bar{h}_{i}^{\{(+)} \gamma_{\mu}\left(1-\gamma^{5}\right) q_{i} \bar{h}_{j}^{(-)\}} \gamma^{\mu}\left(1-\gamma^{5}\right) q_{j}, & \tilde{Q}_{2}=\bar{h}_{i}^{\{(+)}\left(1-\gamma^{5}\right) q_{i} \bar{h}_{j}^{(-)\}}\left(1-\gamma^{5}\right) q_{j}, \\
\tilde{Q}_{4}=\bar{h}_{i}^{\{(+)}\left(1-\gamma^{5}\right) q_{i} \bar{h}_{j}^{(-)\}}\left(1+\gamma^{5}\right) q_{j}, & \tilde{Q}_{5}=\bar{h}_{i}^{\{(+)}\left(1-\gamma^{5}\right) q_{j} \bar{h}_{j}^{(-)\}}\left(1+\gamma^{5}\right) q_{i},
\end{array}
$$

where the HQET field $h^{(+)}(x)$ annihilates a bottom quark, $h^{(-)}(x)$ creates an anti-bottom and we have introduced the notation

$$
\bar{h}^{\{(+)} \Gamma_{A} q \bar{h}^{(-)\}} \Gamma_{B} q=\bar{h}^{(+)} \Gamma_{A} q \bar{h}^{(-)} \Gamma_{B} q+\bar{h}^{(-)} \Gamma_{A} q \bar{h}^{(+)} \Gamma_{B} q .
$$




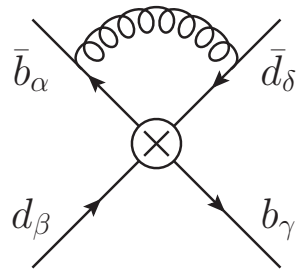

$D_{1}$

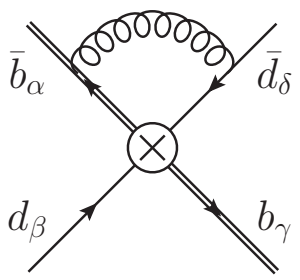

$E_{1}$

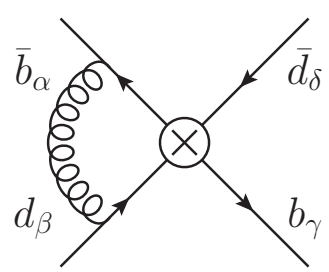

$D_{2}$

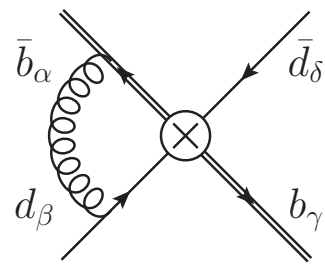

$E_{2}$

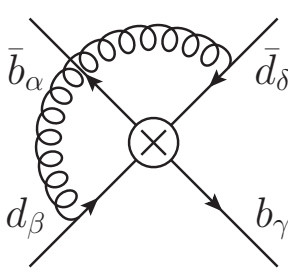

$D_{3}$

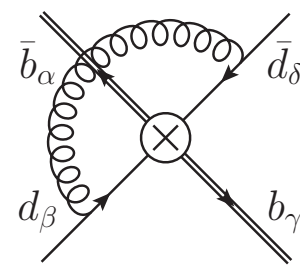

$E_{3}$

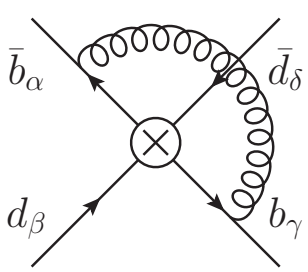

$D_{4}$

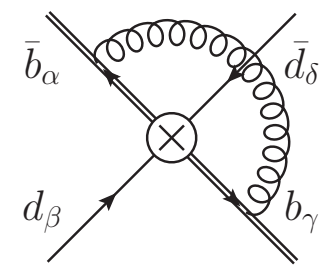

$E_{4}$

Figure 1. QCD $\left(D_{i}\right)$ and HQET $\left(E_{i}\right)$ diagrams that enter the matching. Symmetric diagrams are not shown.

Note that no operator $\tilde{Q}_{3}$ appears on the HQET side because it is not linearly independent, just like its QCD equivalent at leading order in $1 / m_{b}$ [40]. We define the evanescent HQET operators up to three constants $a_{i}$ with $i=1,2,3$ which allow us to keep track of the scheme dependence. Again the explicit basis of the evanescent operators can be found in appendix A. The matching condition for the $\Delta B=2$ operators is given by

$$
\left\langle Q_{i}\right\rangle(\mu)=\sum C_{Q_{i} \tilde{Q}_{j}}(\mu)\left\langle\tilde{Q}_{j}\right\rangle(\mu)+\mathcal{O}\left(\frac{1}{m_{b}}\right),
$$

where $\langle A\rangle=\langle\bar{B}|A| B\rangle$. The matching coefficients can be expanded in perturbation theory and take the form

$$
C_{Q_{i} \tilde{Q}_{j}}(\mu)=C_{Q_{i} \tilde{Q}_{j}}^{(0)}+\frac{\alpha_{s}(\mu)}{4 \pi} C_{Q_{i} \tilde{Q}_{j}}^{(1)}(\mu)+\ldots
$$

Thus the matching calculation can be performed with external quark states. The partonic QCD matrix elements are

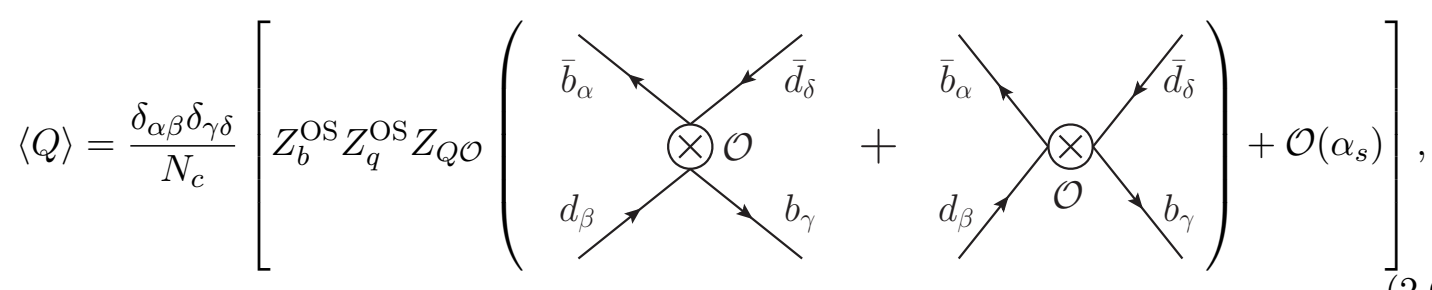

where we sum over $\mathcal{O}$, including all physical and evanescent operators, and the color singlet initial and final state have been projected out. The two tree-level contractions appear with a relative minus sign. The gluon corrections are shown in figure 1 and do not contain self-energy insertions on the external legs, since the quark fields are renormalized in the on-shell scheme. The HQET matrix elements follow from the replacements $Q \rightarrow \tilde{Q}, \mathcal{O} \rightarrow \tilde{\mathcal{O}}$, 
$Z_{b}^{\mathrm{OS}} \rightarrow Z_{h}^{\mathrm{OS}}$ and using HQET propagators instead of the full QCD ones for the bottom quark. The heavy quark on-shell renormalization constants are

$$
Z_{b}^{\mathrm{OS}}=1-\frac{\alpha_{s} C_{F}}{4 \pi}\left(\frac{3}{\epsilon}+4+3 \ln \frac{\mu^{2}}{m_{b}^{2}}\right)+\mathcal{O}\left(\alpha_{s}^{2}\right), \quad Z_{h}^{\mathrm{OS}}=1
$$

The light-quark renormalization is trivial in the massless case $Z_{q}^{\mathrm{OS}}=1$. For the renormalization of the physical operators the $\overline{\mathrm{MS}}$ scheme is used. In accordance with [36-38] the evanescent operators are renormalized by a finite amount such that their physical matrix elements vanish. Consequently the Wilson coefficients $C_{Q_{i} \tilde{E}_{j}}$ are not required for the determination of the hadronic matrix elements and are omitted in the results shown below. However, in the matching computation itself the matrix elements are taken between external on-shell quark states and are therefore not IR finite. While the IR divergences cancel in the matching of the QCD to the HQET operators there are non-vanishing contributions to the physical matching coefficients $C_{Q \tilde{Q}}$ from matrix elements of the evanescent operators that are multiplied by IR poles since the evanescent operators are defined differently in QCD and HQET, cf. appendix A.

We also find that the NLO matching coefficients $C_{Q_{3} \tilde{Q}_{j}}^{(1)}$ of the operator $Q_{3}$ are affected by the finite renormalization of the evanescent operator $\tilde{E}_{2}$ which contains contributions proportional to the physical operators. This usually only happens at NNLO (as is the case for the other operators) but is already present here at NLO because the tree-level matching coefficient $C_{Q_{3} \tilde{E}_{2}}^{(0)}$ of this operator is non-vanishing and, therefore, the NLO matrix element of the evanescent HQET operator $\tilde{E}_{2}$ already appears at NLO in the matching calculation.

In the computation we have used both a manual approach and an automated setup utilizing QGRAF [41] and Mathematica to generate the amplitudes. The Dirac algebra has been performed with a customized version of TRACER [42] as well as with Package-X [43, 44] and the QCD loop integrals have been evaluated using Package-X [43, 44]. We have also checked our results by performing the calculation with a gluon mass as an IR regulator and found full agreement.

\section{$2.2 \quad$ Results}

We write the LO QCD anomalous dimension matrix (ADM) as

$$
\gamma^{(0)}=\left(\begin{array}{cc}
\gamma_{Q Q}^{(0)} & \gamma_{Q E}^{(0)} \\
\gamma_{E Q}^{(0)} & \gamma_{E E}^{(0)}
\end{array}\right)
$$

where $\gamma_{Q Q}^{(0)}$ is the ADM for the physical set of operators (2.1), $\gamma_{Q E}^{(0)}$ describes the mixing of the physical operators into the evanescent ones (A.1), $\gamma_{E Q}^{(0)}$ vanishes (see [38]) and $\gamma_{E E}^{(0)}$ is not required. We decompose the LO HQET ADM $\tilde{\gamma}^{(0)}$ analogously. Our results for the non-vanishing entries are given in appendix A.

The non-vanishing Wilson coefficients at LO are

$$
C_{Q_{1} \tilde{Q}_{1}}^{(0)}=1, \quad C_{Q_{2} \tilde{Q}_{2}}^{(0)}=1, \quad C_{Q_{3} \tilde{Q}_{1}}^{(0)}=-\frac{1}{2}, \quad C_{Q_{3} \tilde{Q}_{2}}^{(0)}=-1, \quad C_{Q_{4} \tilde{Q}_{4}}^{(0)}=1, \quad C_{Q_{5} \tilde{Q}_{5}}^{(0)}=1 .
$$


The NLO corrections to the matching coefficients read

$$
C_{Q \tilde{Q}}^{(1)}=\left(\begin{array}{cccc}
-\frac{41}{3}+\frac{a_{2}}{12}-6 L_{\mu} & -8 & 0 & 0 \\
\frac{3}{2}-\frac{a_{1}}{12}+L_{\mu} & 8+4 L_{\mu} & 0 & 0 \\
5+\frac{2 a_{1}-a_{2}}{24}+4 L_{\mu} & 4+4 L_{\mu} & 0 & 0 \\
0 & 0 & 8-\frac{a_{3}}{24}+\frac{9 L_{\mu}}{2}-4+\frac{a_{3}}{8}-\frac{3 L_{\mu}}{2} \\
0 & 0 & 4+\frac{a_{3}}{8}+\frac{3 L_{\mu}}{2}-8-\frac{a_{3}}{24}-\frac{9 L_{\mu}}{2}
\end{array}\right),
$$

where $L_{\mu}=\ln \left(\mu^{2} / m_{b}^{2}\right)$ and we have set $N_{c}=3$ to keep the results compact.

\subsection{Matching of QCD and HQET Bag parameters}

We define the QCD bag parameters $B_{Q}$ following [45]

$$
\langle Q(\mu)\rangle=A_{Q} f_{B}^{2} M_{B}^{2} B_{Q}(\mu),
$$

where the coefficients read

$$
\begin{array}{ll}
A_{Q_{1}}=2+\frac{2}{N_{c}}, & \\
A_{Q_{2}}=\frac{M_{B}^{2}}{\left(m_{b}+m_{q}\right)^{2}}\left(-2+\frac{1}{N_{c}}\right), & A_{Q_{3}}=\frac{M_{B}^{2}}{\left(m_{b}+m_{q}\right)^{2}}\left(1-\frac{2}{N_{c}}\right), \\
A_{Q_{4}}=\frac{2 M_{B}^{2}}{\left(m_{b}+m_{q}\right)^{2}}+\frac{1}{N_{c}}, & A_{Q_{5}}=1+\frac{2 M_{B}^{2}}{N_{c}\left(m_{b}+m_{q}\right)^{2}},
\end{array}
$$

the $B$ meson decay constant $f_{B}$ is defined as

$$
\left\langle 0\left|\bar{b} \gamma^{\mu} \gamma^{5} q\right| B(p)\right\rangle=-i f_{B} p^{\mu},
$$

$M_{B}$ is the mass of the $B$ meson and $B_{Q_{i}}=1$ corresponds to the VSA approximation. We note that the quark masses appearing in (2.12) are not $\overline{\mathrm{MS}}$ masses which is the usual convention today $[5,46]$, but pole masses. We prefer the definition $(2.11)$ for the analysis because the use of $\overline{\mathrm{MS}}$ masses makes the LO ADM of the Bag parameters explicitly $\mu$ dependent and prohibits an analytic solution of the RGE. At the end we convert our results to the convention of $[5,46]$ which we denote as

$$
\langle Q(\mu)\rangle=\bar{A}_{Q}(\mu) f_{B}^{2} M_{B}^{2} \bar{B}_{Q}(\mu),
$$

where the $\bar{A}_{Q}(\mu)$ follow from $A_{Q}$ with the replacements $m_{b} \rightarrow \bar{m}_{b}(\mu)$ and $m_{q} \rightarrow \bar{m}_{q}(\mu)$. Similar to (2.11), we use for the HQET operators

$$
\langle\tilde{Q}(\mu)\rangle=A_{\tilde{Q}} F^{2}(\mu) B_{\tilde{Q}}(\mu),
$$

where

$$
A_{\tilde{Q}_{1}}=2+\frac{2}{N_{c}}, \quad A_{\tilde{Q}_{2}}=-2+\frac{1}{N_{c}}, \quad A_{\tilde{Q}_{4}}=2+\frac{1}{N_{c}}, \quad A_{\tilde{Q}_{5}}=1+\frac{2}{N_{c}},
$$

and the matrix elements have been taken between non-relativistically normalized states $\left\langle\tilde{Q}_{i}(\mu)\right\rangle \equiv\left\langle\overline{\mathbf{B}}\left|\tilde{Q}_{i}(\mu)\right| \mathbf{B}\right\rangle$ with

$$
|B(p)\rangle=\sqrt{2 M_{B}}|\mathbf{B}(v)\rangle+\mathcal{O}\left(1 / m_{b}\right),
$$


such that

$$
\left\langle\mathbf{B}\left(v^{\prime}\right) \mid \mathbf{B}(v)\right\rangle=\frac{v^{0}}{M_{B}^{3}}(2 \pi)^{3} \delta^{(3)}\left(\mathbf{v}^{\prime}-\mathbf{v}\right) .
$$

The parameter $F(\mu)$ is defined as

$$
\left\langle 0\left|\bar{h}^{(-)} \gamma^{\mu} \gamma^{5} q\right| \mathbf{B}(v)\right\rangle=-i F(\mu) v^{\mu},
$$

and related to the decay constant by

$$
f_{B}=\sqrt{\frac{2}{M_{B}}} C(\mu) F(\mu)+\mathcal{O}\left(1 / m_{b}\right),
$$

with [47]

$$
C(\mu)=1-2 C_{F} \frac{\alpha_{s}(\mu)}{4 \pi}+\mathcal{O}\left(\alpha_{s}^{2}\right)
$$

From (2.11) and (2.15), we obtain, using (2.4), (2.17) and (2.20),

$$
B_{Q_{i}}(\mu)=\sum_{j} \frac{A_{\tilde{Q}_{j}}}{A_{Q_{i}}} \frac{C_{Q_{i} \tilde{Q}_{j}}(\mu)}{C^{2}(\mu)} B_{\tilde{Q}_{j}}(\mu)+\mathcal{O}\left(1 / m_{b}\right) .
$$

The HQET bag parameters $B_{\tilde{Q}}$ are determined from a sum rule analysis.

\section{HQET sum rule}

The HQET sum rule is introduced in section 3.1. We give results for the doublediscontinuity of the three-point correlators in section 3.2 and describe the determination of HQET and QCD Bag parameters in section 3.3.

\subsection{The sum rule}

We define the three-point correlator

$$
K_{\tilde{Q}}\left(\omega_{1}, \omega_{2}\right)=\int d^{d} x_{1} d^{d} x_{2} e^{i p_{1} \cdot x_{1}-i p_{2} \cdot x_{2}}\left\langle 0\left|\mathrm{~T}\left[\tilde{j}_{+}\left(x_{2}\right) \tilde{Q}(0) \tilde{j}_{-}\left(x_{1}\right)\right]\right| 0\right\rangle,
$$

where $\omega_{1,2}=p_{1,2} \cdot v$ and

$$
\tilde{j}_{+}=\bar{q} \gamma^{5} h^{(+)}, \quad \tilde{j}_{-}=\bar{q} \gamma^{5} h^{(-)},
$$

are interpolating currents for the pseudoscalar $\bar{B}$ and $B$ mesons. The correlator (3.1) is analytic in $\omega_{1,2}$ apart from discontinuities for positive real $\omega$. This allows us to construct a dispersion relation

$$
K_{\tilde{Q}}\left(\omega_{1}, \omega_{2}\right)=\int_{0}^{\infty} d \eta_{1} d \eta_{2} \frac{\rho_{\tilde{Q}}\left(\eta_{1}, \eta_{2}\right)}{\left(\eta_{1}-\omega_{1}\right)\left(\eta_{2}-\omega_{2}\right)}+[\text { subtraction terms }],
$$

where $\rho_{\tilde{Q}}$ is the double discontinuity of $K_{\tilde{Q}}$ in $\omega_{1}$ and $\omega_{2}$. The second term on the right originates from the integration of $K_{\tilde{Q}}$ along the circle at infinity in the complex $\eta_{1}$ or 
(and) $\eta_{2}$ planes and is therefore polynomial in $\omega_{1}$ or (and) $\omega_{2}$. The correlator $K_{\tilde{Q}}$ can be computed by means of an OPE

$$
K_{\tilde{Q}}^{\mathrm{OPE}}\left(\omega_{1}, \omega_{2}\right)=K_{\tilde{Q}}^{\mathrm{pert}}\left(\omega_{1}, \omega_{2}\right)+K_{\tilde{Q}}^{\langle\bar{q} q\rangle}\left(\omega_{1}, \omega_{2}\right)\langle\bar{q} q\rangle+K_{\tilde{Q}}^{\left\langle\alpha_{s} G^{2}\right\rangle}\left(\omega_{1}, \omega_{2}\right)\left\langle\alpha_{s} G^{2}\right\rangle+\ldots
$$

for values of $\omega_{1,2}$ that lie far away from the physical cut. Assuming quark-hadron duality, we can equate the correlator $K_{\tilde{Q}}^{\mathrm{OPE}}$ with its hadronic counterpart

$$
K_{\tilde{Q}}^{\mathrm{had}}\left(\omega_{1}, \omega_{2}\right)=\int_{0}^{\infty} d \eta_{1} d \eta_{2} \frac{\rho_{\tilde{Q}}^{\mathrm{had}}\left(\eta_{1}, \eta_{2}\right)}{\left(\eta_{1}-\omega_{1}\right)\left(\eta_{2}-\omega_{2}\right)}+[\text { subtraction terms }]
$$

which is obtained from integration over the hadronic spectral function

$$
\rho_{\tilde{Q}}^{\mathrm{had}}\left(\omega_{1}, \omega_{2}\right)=F^{2}(\mu)\langle\tilde{Q}(\mu)\rangle \delta\left(\omega_{1}-\bar{\Lambda}\right) \delta\left(\omega_{2}-\bar{\Lambda}\right)+\rho_{\tilde{Q}}^{\mathrm{cont}}\left(\omega_{1}, \omega_{2}\right) .
$$

We use a double Borel transformation with respect to $\omega_{1,2}$ to remove the contribution from the integration over the circle at infinity and to suppress the sensitivity to the continuum part $\rho_{\tilde{Q}}^{\text {cont }}$ of the spectral function, which yields the sum rule

$$
\int_{0}^{\infty} d \omega_{1} d \omega_{2} e^{-\frac{\omega_{1}}{t_{1}}-\frac{\omega_{2}}{t_{2}}} \rho_{\tilde{Q}}^{\mathrm{OPE}}\left(\omega_{1}, \omega_{2}\right)=\int_{0}^{\infty} d \omega_{1} d \omega_{2} e^{-\frac{\omega_{1}}{t_{1}}-\frac{\omega_{2}}{t_{2}}} \rho_{\tilde{Q}}^{\mathrm{had}}\left(\omega_{1}, \omega_{2}\right) .
$$

In principle one can proceed by modelling the continuum $\rho_{\tilde{Q}}^{\text {cont }}$. The desired matrix element of the operator $\tilde{Q}$ between the mesonic ground state can then be disentangled by varying the Borel parameters. However, the continuum contribution is exponentially suppressed in the Borel sum rule and it is safe to simply "cut off" the sum rule by assuming that

$$
\rho_{\tilde{Q}}^{\text {cont }}\left(\omega_{1}, \omega_{2}\right)=\rho_{\tilde{Q}}^{\mathrm{OPE}}\left(\omega_{1}, \omega_{2}\right)\left[1-\theta\left(\omega_{c}-\omega_{1}\right) \theta\left(\omega_{c}-\omega_{2}\right)\right],
$$

which directly yields a finite-energy sum rule for the matrix elements

$$
F^{2}(\mu)\langle\tilde{Q}(\mu)\rangle e^{-\frac{\bar{\Lambda}}{t_{1}}-\frac{\bar{\Lambda}}{t_{2}}}=\int_{0}^{\omega_{c}} d \omega_{1} d \omega_{2} e^{-\frac{\omega_{1}}{t_{1}}-\frac{\omega_{2}}{t_{2}}} \rho_{\tilde{Q}}^{\mathrm{OPE}}\left(\omega_{1}, \omega_{2}\right) .
$$

Thus, the determination of the HQET Bag parameters requires the computation of the spectral functions $\rho_{\tilde{Q}}^{\mathrm{OPE}}$. The leading condensate corrections have been determined in $[12,13]$. We compute the $\mathcal{O}\left(\alpha_{s}\right)$ corrections to the perturbative contribution below.

\subsection{Spectral functions at NLO}

We determine the spectral functions by first computing the correlator

$$
K_{\tilde{Q}}^{\mathrm{pert}}\left(\omega_{1}, \omega_{2}\right)=K_{\tilde{Q}}^{(0)}\left(\omega_{1}, \omega_{2}\right)+\frac{\alpha_{s}}{4 \pi} K_{\tilde{Q}}^{(1)}\left(\omega_{1}, \omega_{2}\right)+\ldots
$$




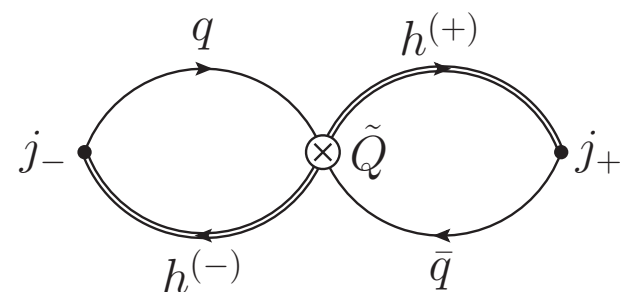

Figure 2. Leading order diagram for the three-point HQET correlator (3.1). The sum over the two possible contractions of the operator $\tilde{Q}$ is implied.
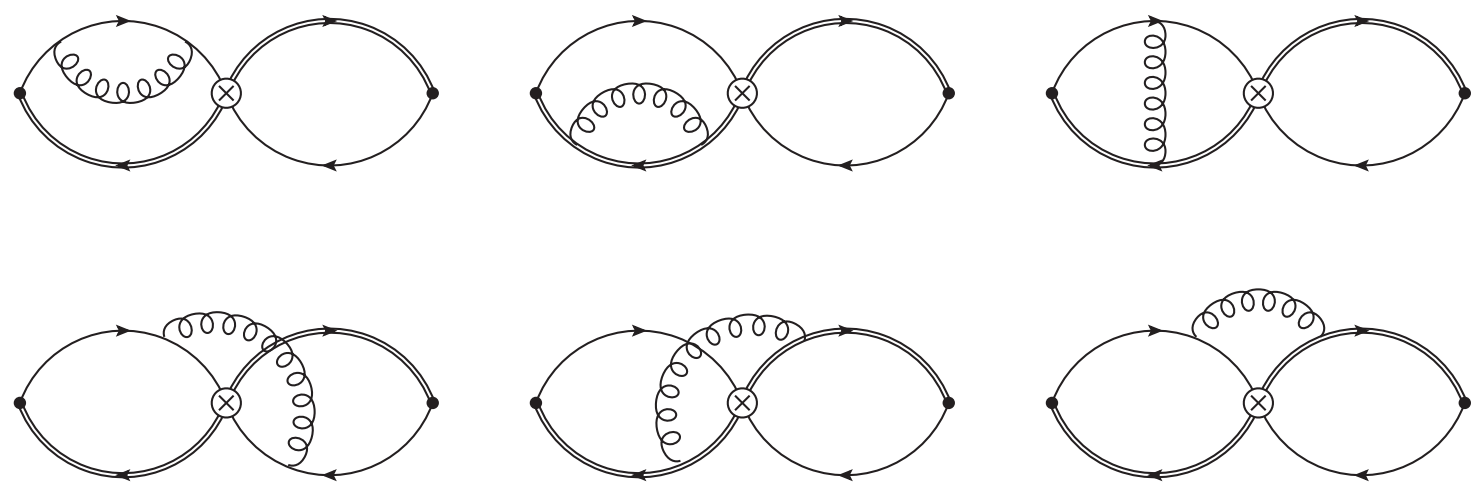

Figure 3. Diagrams contributing to the three-point HQET correlator (3.1) at NLO. Symmetric diagrams are not shown.

and then taking its double discontinuity. At LO we have to evaluate the diagram in figure 2 which factorizes into two two-point functions. We obtain ${ }^{2}$

$$
K_{\tilde{Q}_{i}}^{(0)}\left(\omega_{1}, \omega_{2}\right)=\left(A_{\tilde{Q}_{i}}-\delta_{i 1} \frac{2 \epsilon}{N_{c}}\right) \Pi^{(0)}\left(\omega_{1}\right) \Pi^{(0)}\left(\omega_{2}\right),
$$

where

$$
\Pi^{(0)}(\omega)=-\frac{4 N_{c}}{(4 \pi)^{2-\epsilon}} \tilde{\mu}^{2 \epsilon}(-2 \omega)^{2-2 \epsilon} \Gamma(2-\epsilon) \Gamma(-2+2 \epsilon)
$$

is the LO result for the two-point correlator

$$
\Pi(\omega)=i \int d^{d} x e^{i p x}\left\langle 0\left|\mathrm{~T}\left[\tilde{j}_{+}^{\dagger}(0) \tilde{j}_{+}(x)\right]\right| 0\right\rangle,
$$

where $\omega=p \cdot v$ and the use of $\tilde{\mu}^{2}=\mu^{2} \exp \left(\gamma_{E}\right) /(4 \pi)$ corresponds to the $\overline{\mathrm{MS}}$ scheme.

The bare NLO correction $K_{\tilde{Q}}^{(1), \text { bare }}$ is given by the diagrams shown in figure 3 . At this order we get corrections that do not factorize due to gluon exchange between the left and right-hand side. These genuine three-loop contributions - given by the diagrams in the second row of figure 3 - are the most computationally challenging. The Dirac traces

\footnotetext{
${ }^{2}$ As discussed below the sum rule reproduces the VSA at LO. Therefore the factors $A_{\tilde{Q}_{i}}$ appear at leading order in the expansion of the results in $\epsilon$. However, the correlator is computed in $d$ dimensions and corrections can appear. We find that this happens only for $\tilde{Q}_{1}$ where the contraction of the two $\gamma$ matrices inside the trace yields a $d$-dimensional factor.
} 
have been evaluated with both TRACER [42] and Package-X [43, 44]. We use the code FIRE [48-50] to find IBP relations [51] between the three-loop integrals and to reduce them to a set of master integrals via the Laporta algorithm [52]. The relevant master integrals have been computed analytically in [11,53].

The renormalized NLO correlators are given by

$$
K_{\tilde{Q}_{i}}^{(1)}=K_{\tilde{Q}_{i}}^{(1), \text { bare }}+\frac{1}{2 \epsilon}\left[\left(2 \tilde{\gamma}_{\tilde{j}}^{(0)} \delta_{i j}+\tilde{\gamma}_{\tilde{Q}_{i} \tilde{Q}_{j}}^{(0)}\right) K_{\tilde{Q}_{j}}^{(0)}+\tilde{\gamma}_{\tilde{Q}_{i} \tilde{E}_{j}}^{(0)} K_{\tilde{E}_{j}}^{(0)}\right],
$$

where $\tilde{\gamma}_{\tilde{j}}^{(0)}=-3 C_{F}$ is the LO anomalous dimension of the currents $\tilde{j}_{ \pm}$. The contributions from the evanescent operators modify the double discontinuities of the correlators by a finite amount and introduce a dependence of the correlator on the choice of basis of the HQET evanescent operators. This dependence propagates to the HQET bag parameters extracted in the sum rule and cancels with the HQET evanescent scheme dependence of the matching coefficients (2.10) in the matching equation (2.22) for the QCD Bag parameters. The results for the bare correlators are available as an ancillary Mathematica file with the arXiv version of this article. Here, we only show the compact results for the double discontinuities of the correlators.

Methods to compute the double discontinuities of the correlators have been described in $[11,54]$. The results take the form

$$
\rho_{\tilde{Q}_{i}}^{\text {pert }}\left(\omega_{1}, \omega_{2}\right)=A_{\tilde{Q}_{i}} \rho_{\Pi}\left(\omega_{1}\right) \rho_{\Pi}\left(\omega_{2}\right)+\Delta \rho_{\tilde{Q}_{i}},
$$

where

$$
\begin{aligned}
\rho_{\Pi}(\omega) & \equiv \frac{\Pi(\omega+i 0)-\Pi(\omega-i 0)}{2 \pi i} \\
& =\frac{N_{c} \omega^{2}}{2 \pi^{2}}\left[1+\frac{\alpha_{s} C_{F}}{4 \pi}\left(17+\frac{4 \pi^{2}}{3}+3 \ln \frac{\mu^{2}}{4 \omega^{2}}\right)+\mathcal{O}\left(\alpha_{s}^{2}\right)\right],
\end{aligned}
$$

is the discontinuity of the two-point correlator (3.13) up to two-loop order [55-57]. The non-factorizable contributions are

$$
\Delta \rho_{\tilde{Q}_{i}} \equiv \frac{\omega_{1}^{2} \omega_{2}^{2}}{\pi^{4}} \frac{\alpha_{s}}{4 \pi} r_{\tilde{Q}_{i}}\left(x, L_{\omega}\right),
$$

where $x=\omega_{2} / \omega_{1}, L_{\omega}=\ln \left(\mu^{2} /\left(4 \omega_{1} \omega_{2}\right)\right)$ and we obtain

$$
\begin{aligned}
& r_{\tilde{Q}_{1}}\left(x, L_{\omega}\right)=8-\frac{a_{2}}{2}-\frac{8 \pi^{2}}{3}, \\
& r_{\tilde{Q}_{2}}\left(x, L_{\omega}\right)=25+\frac{a_{1}}{2}-\frac{4 \pi^{2}}{3}+6 L_{\omega}+\phi(x), \\
& r_{\tilde{Q}_{4}}\left(x, L_{\omega}\right)=16-\frac{a_{3}}{4}-\frac{4 \pi^{2}}{3}+3 L_{\omega}+\frac{\phi(x)}{2}, \\
& r_{\tilde{Q}_{5}}\left(x, L_{\omega}\right)=29-\frac{a_{3}}{2}-\frac{8 \pi^{2}}{3}+6 L_{\omega}+\phi(x),
\end{aligned}
$$

where

$$
\phi(x)= \begin{cases}x^{2}-8 x+6 \ln (x), & x \leq 1 \\ \frac{1}{x^{2}}-\frac{8}{x}-6 \ln (x), & x>1 .\end{cases}
$$

Taking $a_{2}=-4$ in accordance with [11] we reproduce their result for $r_{\tilde{Q}_{1}}$ up to a factor of 2 which is due to the different normalization of the HQET operators. 


\subsection{Sum rule for the Bag parameters}

Inserting the decomposition (3.15) into the sum rule (3.9) allows us to subtract the factorized contribution using the sum rule [55-57] for the HQET decay constant

$$
F^{2}(\mu) e^{-\frac{\bar{\Lambda}}{t}}=\int_{0}^{\omega_{c}} d \omega e^{-\frac{\omega}{t}} \rho_{\Pi}(\omega)+\ldots
$$

The factorizable part of (3.15) exactly reproduces the VSA for the matrix elements. After subtracting it, we obtain a sum rule for the deviation $\Delta B_{\tilde{Q}}=B_{\tilde{Q}}-1$ from the VSA. In the traditional sum rule approach this gives

$$
\begin{aligned}
\Delta B_{\tilde{Q}_{i}} & =\frac{1}{A_{\tilde{Q}_{i}} F(\mu)^{4}} \int_{0}^{\omega_{c}} d \omega_{1} d \omega_{2} e^{\frac{\bar{\Lambda}-\omega_{1}}{t_{1}}+\frac{\bar{T}-\omega_{2}}{t_{2}}} \Delta \rho_{\tilde{Q}_{i}}\left(\omega_{1}, \omega_{2}\right) \\
& =\frac{1}{A_{\tilde{Q}_{i}}} \frac{\int_{0}^{\omega_{c}} d \omega_{1} d \omega_{2} e^{-\frac{\omega_{1}}{t_{1}}-\frac{\omega_{2}}{t_{2}}} \Delta \rho_{\tilde{Q}_{i}}\left(\omega_{1}, \omega_{2}\right)}{\left(\int_{0}^{\omega_{c}} d \omega_{1} e^{-\frac{\omega_{1}}{t_{1}}} \rho_{\Pi}\left(\omega_{1}\right)\right)\left(\int_{0}^{\omega_{c}} d \omega_{2} e^{-\frac{\omega_{2}}{t_{2}}} \rho_{\Pi}\left(\omega_{2}\right)\right)} .
\end{aligned}
$$

The stability of the sum rule (3.22) can then be assessed numerically by variation of the cutoff $\omega_{c}$ and the Borel parameters $t_{i}$, see e.g. [54, 56].

In our analysis we follow a different approach that allows us to obtain analytic results for the HQET Bag parameters. This exploits the fact that the dispersion relation (3.3) is not violated by the introduction of an arbitrary weight function $w\left(\omega_{1}, \omega_{2}\right)$ in the integration as long as it is chosen such that no additional discontinuities appear in the complex plane. ${ }^{3}$ In the presence of such a weight function $w$ the square of the sum rule (3.20) takes the form

$$
F^{4}(\mu) e^{-\frac{\bar{\Lambda}}{t_{1}}-\frac{\bar{\Lambda}}{t_{2}}} w(\bar{\Lambda}, \bar{\Lambda})=\int_{0}^{\omega_{c}} d \omega_{1} d \omega_{2} e^{-\frac{\omega_{1}}{t_{1}}-\frac{\omega_{2}}{t_{2}}} w\left(\omega_{1}, \omega_{2}\right) \rho_{\Pi}\left(\omega_{1}\right) \rho_{\Pi}\left(\omega_{2}\right)+\ldots
$$

Since the condensate contributions have already been taken into account in [11-13] and are in the subpercent range we only focus on the perturbative contribution to the sum rule. By using (3.23) with the choice

$$
w_{\tilde{Q}_{i}}\left(\omega_{1}, \omega_{2}\right)=\frac{\Delta \rho_{\tilde{Q}_{i}}^{\text {pert }}\left(\omega_{1}, \omega_{2}\right)}{\rho_{\Pi}^{\text {pert }}\left(\omega_{1}\right) \rho_{\Pi}^{\text {pert }}\left(\omega_{2}\right)}=\frac{4}{N_{c}^{2}} \frac{\alpha_{s}}{4 \pi} r_{\tilde{Q}_{i}}\left(x, L_{\omega}\right),
$$

we can remove the integration in (3.21) altogether and find the simple result

$$
\Delta B_{\tilde{Q}_{i}}^{\text {pert }}\left(\mu_{\rho}\right)=\frac{4}{N_{c}^{2} A_{\tilde{Q}_{i}}} \frac{\alpha_{s}\left(\mu_{\rho}\right)}{4 \pi} r_{\tilde{Q}_{i}}\left(1, \log \frac{\mu_{\rho}^{2}}{4 \bar{\Lambda}^{2}}\right) .
$$

\footnotetext{
${ }^{3}$ The arbitrariness of the weight function is a mathematical statement which holds for the dispersion relation. The sum rule (3.7) does however also assume quark-hadron duality and breaks down if pathological weight functions are used, e.g. rapidly oscillating ones. In the following we only use slowly varying weight functions with support on the complete integration domain.
} 
The sum rule is valid at a low scale $\mu_{\rho} \sim 2 \omega_{i} \sim 2 \bar{\Lambda}$ where the logarithms that appear in the spectral functions are small. From there we have to evolve the results for the Bag parameters up to the scale $\mu_{m} \sim m_{b}$ where the matching (2.22) to the QCD Bag parameters can be performed without introducing large logarithms. From (2.15) and the running of the HQET operators and decay constant

$$
\frac{d \overrightarrow{\tilde{Q}}}{d \ln \mu}=-\hat{\tilde{\gamma}}_{\tilde{Q} \tilde{Q}} \overrightarrow{\tilde{Q}}, \quad \frac{d F(\mu)}{d \ln \mu}=-\tilde{\gamma}_{\tilde{j}} F(\mu),
$$

we obtain the RG equations for the HQET Bag parameters

$$
\frac{d \vec{B}_{\tilde{Q}}}{d \ln \mu}=-\left(\hat{A}_{\tilde{Q}}^{-1} \hat{\tilde{\gamma}}_{\tilde{Q} \tilde{Q}} \hat{A}_{\tilde{Q}}-2 \tilde{\gamma}_{\tilde{j}}\right) \vec{B}_{\tilde{Q}} \equiv-\hat{\tilde{\gamma}}_{\tilde{B}} \vec{B}_{\tilde{Q}}
$$

where $\hat{A}_{\tilde{Q}}$ is the diagonal matrix with entries $A_{\tilde{Q}}$ given in (2.16). The LO solution to (3.27) takes the form

$$
\vec{B}_{\tilde{Q}}(\mu)=\hat{U}_{\tilde{B}}^{(0)}\left(\mu, \mu_{0}\right) \vec{B}_{\tilde{Q}}\left(\mu_{0}\right),
$$

with the LO evolution matrix

$$
\hat{U}_{\tilde{B}}^{(0)}\left(\mu, \mu_{0}\right)=\left(\frac{\alpha_{s}(\mu)}{\alpha_{s}\left(\mu_{0}\right)}\right)^{\frac{\hat{\gamma}_{\tilde{B}}^{(0)}}{2 \beta_{0}}}=\hat{V}\left(\frac{\alpha_{s}(\mu)}{\alpha_{s}\left(\mu_{0}\right)}\right)^{\frac{\vec{\gamma}_{\tilde{B}}^{(0)}}{2 \beta_{0}}} \hat{V}^{-1}
$$

where $\hat{V}$ is the transformation that diagonalizes the $\operatorname{ADM} \hat{\tilde{\gamma}}_{\tilde{B}}^{(0)}$

$$
\hat{\tilde{\gamma}}_{\tilde{B}}^{(0), \mathrm{D}}=\hat{V}^{-1} \hat{\tilde{\gamma}}_{\tilde{B}}^{(0)} \hat{V},
$$

and the vector $\overrightarrow{\tilde{\gamma}}_{\tilde{B}}^{(0)}$ contains the diagonal entries of $\hat{\tilde{\gamma}}_{\tilde{B}}^{(0), \mathrm{D}}$. As part of our error analysis we allow the matching scale $\mu_{m}$ to differ from $\bar{m}_{b}\left(\bar{m}_{b}\right)$ and then evolve the QCD Bag parameters back to $\bar{m}_{b}\left(\bar{m}_{b}\right)$. The LO evolution matrix has the same form as its HQET counterpart (3.29) while the anomalous dimension matrix of the QCD Bag parameters is given by

$$
\hat{\gamma}_{B}=\hat{A}_{Q}^{-1} \hat{\gamma}_{Q Q} \hat{A}_{Q}
$$

We only resum the leading logarithms because the NLO anomalous dimensions in HQET are currently not known. This implies that dependence of the QCD matrix elements on the basis of evanescent HQET operators does not fully cancel. As discussed below, we use variation of the parameters $a_{i}$ to estimate the effects of NLL resummation. We expect this effect to be small since the scales $\mu_{\rho}$ and $\mu_{m}$ are not very widely separated and $\ln \left(\mu_{m} / \mu_{\rho}\right)$ is of order one.

\section{Results for $\Delta B=2$ operators}

We describe our analysis in section 4.1 and give the results for the Bag parameters, together with a comparison with other works, in section 4.2. In section 4.3 the results for the mixing observables with our Bag parameters are shown. 


\subsection{Details of the analysis}

We determine the HQET Bag parameters from the sum rule (3.25) with the central values $\mu_{\rho}=1.5 \mathrm{GeV}$ and $\bar{\Lambda}=0.5 \mathrm{GeV}$. We use RunDec [58, 59] to evolve $\alpha_{s}\left(M_{Z}\right)=0.1181$ [60] down to the bottom-quark $\overline{\mathrm{MS}}$ mass $\bar{m}_{b}\left(\bar{m}_{b}\right)=4.203 \mathrm{GeV}[61,62]$ with five-loop accuracy [63-67]. From there we use two-loop running with four and five flavours in HQET and QCD, respectively. The decoupling of the bottom quark is trivial at this accuracy.

The HQET Bag parameters are then evolved from the scale $\mu_{\rho}$ up to the scale $\mu_{m}=$ $\bar{m}_{b}\left(\bar{m}_{b}\right)$ using (3.28). There the matching (2.22) to the QCD Bag parameters is performed. The factors $C_{Q_{i} \tilde{Q}_{j}}(\mu) / C^{2}(\mu)$ are expanded in $\alpha_{s}$ and truncated after the linear term. We also expand the ratios $A_{\tilde{Q}_{j}} / A_{Q_{i}}$ strictly in $\bar{\Lambda} / m_{b}$ and $m_{q} / m_{b}$. Up to higher order perturbative corrections, this is equivalent to the use of the VSA for the power-suppressed HQET operators that arise in the QCD-HQET matching (2.4).

A small dependence on the choice of basis for the evanescent HQET operators remains in the QCD Bag parameters because the RG evolution of the HQET Bag parameters is only known at the LL level. We have checked that the $a_{i}$-dependence fully cancels when the scales $\mu_{\rho}$ and $\mu_{m}$ are identified and the matching (2.22) is strictly expanded in the strong coupling, which serves as a strong cross-check of our calculation. For different scales $\mu_{\rho}$ and $\mu_{m}$ the remaining $a_{i}$-dependence can be removed by a future computation of the NLO ADMs.

Finally, we convert the QCD Bag parameters $B_{Q}$ to the usual convention $\bar{B}_{Q}$ defined in (2.14). This is done by expanding the ratios of the prefactors $A_{Q} / \bar{A}_{Q}\left(\bar{m}_{b}\left(\bar{m}_{b}\right)\right)$ in $\alpha_{s}$ and truncating them after the linear term.

To estimate the errors of the Bag parameters we take the following sources of uncertainties into account:

- The uncertainty in the analytic form (3.25) of the sum rule is estimated through variation of the residual mass $\bar{\Lambda}$ in the range $[0.4,0.6] \mathrm{GeV}$. In addition we include an intrinsic sum rule uncertainty of 0.02 in the HQET bag parameters. The numerical value is determined from the comparison of the analytic values (3.25) with results obtained from the traditional sum rule approach (3.22).

- The condensate contributions to $B_{\tilde{Q}_{1}}$ and $B_{\tilde{Q}_{2}}$ are taken from $[12,13]$ and are in the subpercent range. For $B_{\tilde{Q}_{4}}$ and $B_{\tilde{Q}_{5}}$, which have not been determined there, we therefore add an error of \pm 0.01 to the perturbative results.

- To assign an uncertainty from the unknown $\alpha_{s}^{2}$ contributions to the spectral densities we vary the scale $\mu_{\rho}$ in the range $[1,2] \mathrm{GeV}$.

- As discussed above we implicitly include higher-order corrections in $1 / m_{b}$ in the VSA approximation. The non-factorizable corrections of this kind are of the order $\left(\alpha_{s} / \pi\right) \cdot\left(\bar{\Lambda} / m_{b}\right) \sim 0.01$, which we take as an estimate for the error.

- Higher order perturbative contributions to the QCD-HQET matching relation and the RG evolution of the Bag parameters are estimated through variation of $\mu_{m}$ in 
the range $[3,6] \mathrm{GeV}$ and variation of the $a_{i}$ in the range $[-10,10]$. The QCD Bag parameters are then evolved to the central scale $\bar{m}_{b}\left(\bar{m}_{b}\right)$ with LL accuracy as described in section 3.3.

The variation of $\mu_{m}$ by the usual factors of $1 / 2$ and 2 would lead to a doubling of the matching uncertainty estimates given below, which would significantly exceed the effect of the NLO matching at the central scale. We therefore use a less conservative range but cannot exclude larger matching effects at NNLO at present, while a calculation is not available.

- The parametric uncertainty from $\alpha_{s}\left(M_{Z}\right)$ is in the permille range and neglected.

The individual errors are then summed in quadrature. We also divide the uncertainties into a sum rule uncertainty which contains the first three items in the list above and a matching uncertainty which contains the remaining three.

\subsection{Results and comparison}

From the sum rule we obtain the HQET Bag parameters

$$
\begin{aligned}
& \left.\left.B_{\tilde{Q}_{1}}(1.5 \mathrm{GeV})=0.910_{-0.031}^{+0.023}=0.910_{-0.000}^{+0.000}(\bar{\Lambda})_{-0.020}^{+0.020} \text { (intr. }\right)_{-0.005}^{+0.005} \text { (cond. }\right)_{-0.024}^{+0.011}\left(\mu_{\rho}\right), \\
& \left.\left.B_{\tilde{Q}_{2}}(1.5 \mathrm{GeV})=0.923_{-0.035}^{+0.029}=0.923_{-0.020}^{+0.016}(\bar{\Lambda})_{-0.020}^{+0.020} \text { (intr. }\right)_{-0.004}^{+0.004} \text { (cond. }\right)_{-0.020}^{+0.013}\left(\mu_{\rho}\right), \\
& \left.\left.B_{\tilde{Q}_{4}}(1.5 \mathrm{GeV})=1.009_{-0.023}^{+0.024}=1.009_{-0.006}^{+0.007}(\bar{\Lambda})_{-0.020}^{+0.020} \text { (intr. }\right)_{-0.010}^{+0.010} \text { (cond. }\right)_{-0.003}^{+0.003}\left(\mu_{\rho}\right), \\
& \left.\left.B_{\tilde{Q}_{5}}(1.5 \mathrm{GeV})=1.004_{-0.028}^{+0.030}=1.004_{-0.016}^{+0.020}(\bar{\Lambda})_{-0.020}^{+0.020} \text { (intr. }\right)_{-0.010}^{+0.010} \text { (cond. }\right)_{-0.006}^{+0.004}\left(\mu_{\rho}\right),
\end{aligned}
$$

where we have set $a_{i}=0$ for $i=1,2,3$ to specify a unique basis of evanescent HQET operators. The individual uncertainties were determined as described above and added in quadrature. The corrections to the VSA for scales in the range from $1-2 \mathrm{GeV}$ are at the level of $5-11 \%$ for $\tilde{Q}_{1,2}$ and $0-4 \%$ for $\tilde{Q}_{4,5}$. We find that the total sum rule uncertainties of the Bag parameters are quite small. This is because the sum rule (3.25) is formulated for the deviation from the VSA and the substantial relative uncertainties of the sum rule itself are small in comparison with the VSA contribution to the Bag parameters.

Following the steps outlined in section 4.1 we obtain the following results for the QCD Bag parameters

$$
\begin{aligned}
& \bar{B}_{Q_{1}}\left(\bar{m}_{b}\left(\bar{m}_{b}\right)\right)=0.868_{-0.050}^{+0.051}=0.868_{-0.029}^{+0.021} \text { (sum rule) }{ }_{-0.041}^{+0.046} \text { (matching), } \\
& \bar{B}_{Q_{2}}\left(\bar{m}_{b}\left(\bar{m}_{b}\right)\right)=0.842_{-0.073}^{+0.078}=0.842_{-0.033}^{+0.028} \text { (sum rule) }{ }_{-0.065}^{+0.073} \text { (matching), } \\
& \bar{B}_{Q_{3}}\left(\bar{m}_{b}\left(\bar{m}_{b}\right)\right)=0.818_{-0.159}^{+0.162}=0.818_{-0.132}^{+0.126} \text { (sum rule) }{ }_{-0.087}^{+0.102} \text { (matching), } \\
& \bar{B}_{Q_{4}}\left(\bar{m}_{b}\left(\bar{m}_{b}\right)\right)=1.049_{-0.084}^{+0.092}=1.049_{-0.025}^{+0.025}(\text { sum rule })_{-0.080}^{+0.089} \text { (matching), } \\
& \left.\bar{B}_{Q_{5}}\left(\bar{m}_{b}\left(\bar{m}_{b}\right)\right)=1.073_{-0.075}^{+0.083}=1.073_{-0.026}^{+0.028}(\text { sum rule })_{-0.070}^{+0.078} \text { (matching }\right) .
\end{aligned}
$$

The evolution to the scale $\left.\bar{m}_{b}\left(\bar{m}_{b}\right)\right)$ and the matching to QCD increase the deviations from the VSA to up to $18 \%$. With the exception of $\bar{B}_{Q_{3}}$ the uncertainties of the Bag parameters are dominated by the matching. A detailed list of the uncertainties can be found in appendix B. 


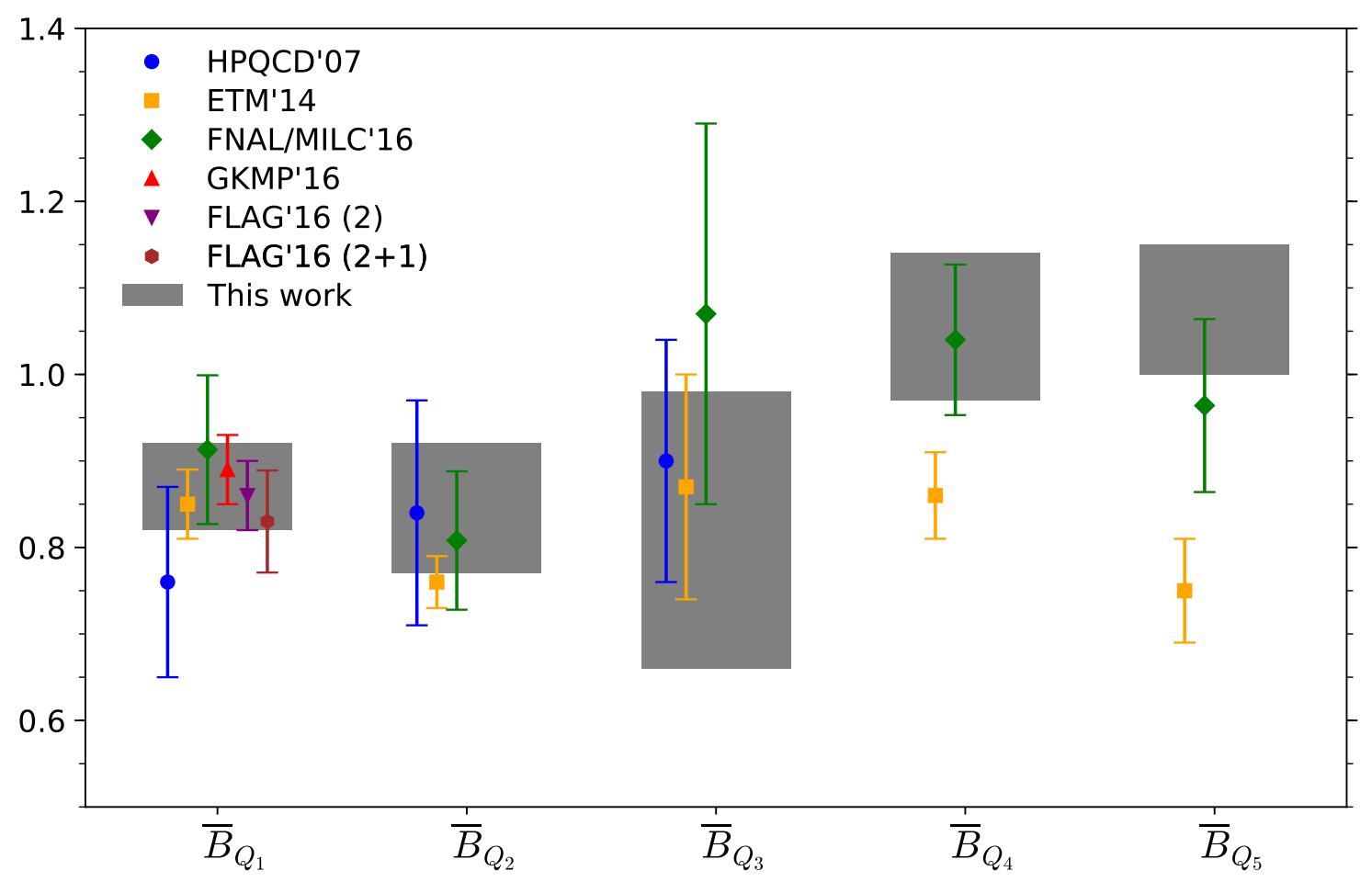

Figure 4. Comparison of our results for the $\Delta B=2$ Bag parameters at the scale $\bar{m}_{b}\left(\bar{m}_{b}\right)$ to the lattice values of HPQCD'07 [3], ETM'14 [4] and FNAL/MILC'16 [5], the FLAG averages [68] and the sum rule result GKMP'16 [11].

In figure 4 we compare our results to other recent determinations from lattice simulations [3-5, 68] and sum rules [11]. We find excellent agreement for the Bag parameters of the operators $Q_{1}, Q_{2}$ and $Q_{3}$. The uncertainties of our sum rule analysis are similar to those obtained on the lattice. We observe that the uncertainty of the Bag parameter $\bar{B}_{Q_{3}}$ is significantly larger than those of $\bar{B}_{Q_{1}}$ and $\bar{B}_{Q_{2}}$. This is related to the small color factor $A_{Q_{3}}=1 / 3+\mathcal{O}\left(1 / m_{b}\right)$ which implies that the sum rule uncertainties get enhanced by the factors $A_{\tilde{Q}_{1}} / A_{Q_{3}}=8+\mathcal{O}\left(1 / m_{b}\right)$ and $A_{\tilde{Q}_{2}} / A_{Q_{3}}=-5+\mathcal{O}\left(1 / m_{b}\right)$ in the matching $(2.22)$ of the Bag parameters. The absolute sum rule uncertainty of the matrix element of $Q_{3}$ is of a similar size as that of the other operators.

The tiny difference of the central value of $\bar{B}_{Q_{1}}$ compared to the sum rule determination [11] is mostly due to different scale choices. Since $\bar{B}_{Q_{1}}$ does not run at the LL order, [11] sets all scales equal to the bottom-quark mass. We, however, evaluate the sum rule at a lower scale $\mu_{\rho} \sim 1.5 \mathrm{GeV}$ where the strong coupling is larger and causes a bigger deviation from the VSA.

Only two previous lattice results $[4,5]$ exist for the matrix elements of the operators $Q_{4}$ and $Q_{5}$, and they differ at the level of more than two sigma. Our results are in very good agreement with those of [5] and show an even higher level of tension with [4] in $\bar{B}_{Q_{5}}$. 


\section{3 $\quad B_{s}$ and $B_{d}$ mixing observables}

We consider the mass and decay rate differences $\Delta M_{s}=M_{H}^{s}-M_{L}^{s}$ and $\Delta \Gamma_{s}=\Gamma_{L}^{s}-\Gamma_{H}^{s}$, where $M_{H / L}^{s}$ and $\Gamma_{H / L}^{s}$ are the mass and width of the heavy $(\mathrm{H})$ and light $(\mathrm{L})$ physical eigenstates of the $B_{s}-\bar{B}_{s}$ system, as well as the semileptonic decay asymmetry

$$
a_{\mathrm{sl}}^{s}=\frac{\Gamma\left(\bar{B}_{s}(t) \rightarrow f\right)-\Gamma\left(B_{s}(t) \rightarrow \bar{f}\right)}{\Gamma\left(\bar{B}_{s}(t) \rightarrow f\right)+\Gamma\left(B_{s}(t) \rightarrow \bar{f}\right)},
$$

where $f$ is a flavor-specific final state, i.e. $\bar{B}_{s} \rightarrow f$ and $B_{s} \rightarrow \bar{f}$ are forbidden (see [1] for a recent review of $B_{s}$ mixing). Using our values for the Bag parameters, we give predictions for these observables and compare them to the current experimental averages given by the HFLAV [69]. In our sum rule determination we have assumed the light quark $q$ in the $B_{q}$ meson to be massless. The corrections to (3.25) from a non-zero strange-quark mass are of the order $\left(\alpha_{s} / \pi\right)\left(m_{s} /(2 \bar{\Lambda})\right) \approx 0.02$. This point has recently been discussed in more detail in [70]. We add another uncertainty of \pm 0.02 in quadrature to the results (4.2) to account for the unknown corrections. The effect on the total uncertainty is small.

We find excellent agreement between experiment and the SM prediction for the mass difference:

$$
\begin{aligned}
& \Delta M_{s}^{\exp }=(17.757 \pm 0.021) \mathrm{ps}^{-1}, \\
& \left.\left.\left.\Delta M_{s}^{\mathrm{SM}}=\left(18.1_{-1.8}^{+1.9}\right) \mathrm{ps}^{-1}=\left(18.1_{-1.2}^{+1.3} \text { (had. }\right) \pm 0.1 \text { (scale }\right)_{-1.3}^{+1.4} \text { (param. }\right)\right) \mathrm{ps}^{-1},
\end{aligned}
$$

where we have used the input values given in appendix B. The $10 \%$ uncertainty of the SM prediction is dominated by the hadronic and parametric CKM uncertainties which are of the same size. We also give results for the mass difference in the $B_{d}$ system

$$
\begin{aligned}
& \Delta M_{d}^{\mathrm{exp}}=(0.5065 \pm 0.0019) \mathrm{ps}^{-1}, \\
& \left.\left.\left.\Delta M_{d}^{\mathrm{SM}}=(0.61 \pm 0.09) \mathrm{ps}^{-1}=(0.61 \pm 0.04 \text { (had. }) \pm 0.00 \text { (scale }\right) \pm 0.08 \text { (param. }\right)\right) \mathrm{ps}^{-1},
\end{aligned}
$$

where the agreement is at the level of 1.1 sigma.

We determine the decay rate difference and the semileptonic decay asymmetry in the $\overline{\mathrm{MS}}$, PS [71], 1S [72] and kinetic [73] mass schemes with the mass values given in appendix B. The $\overline{\mathrm{MS}}$ charm-quark mass at the scale of the bottom-quark mass has been used throughout. We obtain

$$
\begin{aligned}
\Delta \Gamma_{s}^{\mathrm{exp}} & =(0.090 \pm 0.005) \mathrm{ps}^{-1} \\
\Delta \Gamma_{s}^{\overline{\mathrm{MS}}} & =\left(0.080_{-0.023}^{+0.018}\right) \mathrm{ps}^{-1}=\left(0.080 \pm 0.016(\text { had. })_{-0.015}^{+0.006}(\text { scale }) \pm 0.006(\text { param. })\right) \mathrm{ps}^{-1} \\
\Delta \Gamma_{s}^{\mathrm{PS}} & \left.=\left(0.079_{-0.026}^{+0.020}\right) \mathrm{ps}^{-1}=\left(0.079 \pm 0.018(\text { had. })_{-0.018}^{+0.007} \text { (scale }\right) \pm 0.006(\text { param. })\right) \mathrm{ps}^{-1} \\
\Delta \Gamma_{s}^{1 \mathrm{~S}} & \left.\left.\left.=\left(0.075_{-0.028}^{+0.021}\right) \mathrm{ps}^{-1}=(0.075 \pm 0.019 \text { (had. })_{-0.020}^{+0.008} \text { (scale }\right) \pm 0.006 \text { (param. }\right)\right) \mathrm{ps}^{-1} \\
\Delta \Gamma_{s}^{\mathrm{kin}} & \left.\left.\left.=\left(0.076_{-0.027}^{+0.020}\right) \mathrm{ps}^{-1}=(0.076 \pm 0.018 \text { (had. })_{-0.019}^{+0.008} \text { (scale }\right) \pm 0.006 \text { (param. }\right)\right) \mathrm{ps}^{-1}
\end{aligned}
$$

and

$$
\begin{array}{ll}
a_{\mathrm{sl}}^{s, \exp }=(-60 \pm 280) \cdot 10^{-5}, & \\
a_{\mathrm{sl}}^{s, \overline{\mathrm{MS}}}=(2.1 \pm 0.3) \cdot 10^{-5} & =\left(2.1 \pm 0.1(\text { had. })_{-0.1}^{+0.0}(\text { scale })_{-0.3}^{+0.2}(\text { param. })\right) \cdot 10^{-5}, \\
a_{\mathrm{sl}}^{s, \mathrm{PS}}=\left(2.0_{-0.3}^{+0.2}\right) \cdot 10^{-5} & =\left(2.0 \pm 0.1(\text { had. })_{-0.1}^{+0.0}(\mathrm{scale}) \pm 0.2(\text { param. })\right) \cdot 10^{-5}, \\
a_{\mathrm{sl}}^{s, 1 \mathrm{~S}}=\left(2.0_{-0.3}^{+0.2}\right) \cdot 10^{-5} & =\left(2.0 \pm 0.0(\text { had. })_{-0.1}^{+0.0}(\text { scale }) \pm 0.2(\text { param. })\right) \cdot 10^{-5}, \\
a_{\mathrm{sl}}^{s, \mathrm{kin}}=\left(2.0_{-0.3}^{+0.2}\right) \cdot 10^{-5} & =\left(2.0 \pm 0.1(\text { had. })_{-0.1}^{+0.0}(\text { scale }) \pm 0.2(\text { param. })\right) \cdot 10^{-5} .
\end{array}
$$


The different mass schemes are in good agreement with each other and we adopt the PS mass scheme as our central result. The SM value for the decay rate difference is in good agreement with the experimental average. The theory uncertainty is currently at the level of $30 \%$. It is dominated by the matrix elements of the dimension seven operators, in particular the VSA estimate $\bar{B}_{R_{2}}=1 \pm 0.5$ contributes $\pm 0.016 \mathrm{ps}^{-1}$ to the uncertainty. The second largest contribution is the scale variation. A detailed overview is given in appendix B. To achieve a significant reduction of the combined uncertainties, a determination of the dimension seven matrix elements and a NNLO calculation of the perturbative matching are needed.

The experimental uncertainty for the semileptonic decay asymmetry is two orders of magnitude larger than the SM prediction, which makes this a clear null test for the SM [74]. The decay rate difference and the semileptonic decay asymmetry in the $B_{d}$ system have also not been measured yet. The current experimental averages and our predictions are

$$
\begin{aligned}
\Delta \Gamma_{d}^{\exp } & =(-1.3 \pm 6.6) \cdot 10^{-3} \mathrm{ps}^{-1} \\
\Delta \Gamma_{d}^{\mathrm{PS}} & =\left(2.7_{-0.9}^{+0.8}\right) \cdot 10^{-3} \mathrm{ps}^{-1}=\left(2.7_{-0.6}^{+0.6}(\text { had. })_{-0.6}^{+0.2}(\text { scale })_{-0.4}^{+0.4}(\text { param. })\right) \cdot 10^{-3} \mathrm{ps}^{-1}, \\
a_{\mathrm{sl}}^{d, \exp } & =(-21 \pm 17) \cdot 10^{-4}, \\
a_{\mathrm{sl}}^{d, \mathrm{PS}} & =(-4.0 \pm 0.5) \cdot 10^{-4}=\left(-4.0 \pm 0.1(\text { had. })_{-0.1}^{+0.2}(\text { scale }) \pm 0.5(\text { param. })\right) \cdot 10^{-4} .
\end{aligned}
$$

The results obtained in different mass schemes are compatible and the relative uncertainties of the predictions are of the same magnitude as in the $B_{s}$ system.

\section{$5 \Delta B=0$ operators and ratios of $B$-meson lifetimes}

The dominant contribution to lifetime differences between the mesons $B_{q}$ with $q=u, d, s$ is due to spectator effects which first appear as dimension-six contributions in the HQE. The NLO Wilson coefficients have been computed in [75-77]. The dimension seven contributions are known at LO [29, 78]. We define the set of operators in section 5.1 and present the results for their Bag parameters in section 5.2. The updated HQE results for the B-meson lifetime ratios are given in section 5.3.

\subsection{Operators and matrix elements}

The following QCD operators enter at dimension six:

$$
\begin{array}{rlrl}
Q_{1}^{q} & =\bar{b} \gamma_{\mu}\left(1-\gamma^{5}\right) q \bar{q} \gamma^{\mu}\left(1-\gamma^{5}\right) b, & & T_{1}^{q}=\bar{b} \gamma_{\mu}\left(1-\gamma^{5}\right) T^{A} q \bar{q} \gamma^{\mu}\left(1-\gamma^{5}\right) T^{A} b, \\
Q_{2}^{q}=\bar{b}\left(1-\gamma^{5}\right) q \bar{q}\left(1+\gamma^{5}\right) b, & & T_{2}^{q}=\bar{b}\left(1-\gamma^{5}\right) T^{A} q \bar{q}\left(1+\gamma^{5}\right) T^{A} b .
\end{array}
$$

On the HQET side they match onto

$$
\begin{array}{rlrl}
\tilde{Q}_{1}^{q} & =\bar{h} \gamma_{\mu}\left(1-\gamma^{5}\right) q \bar{q} \gamma^{\mu}\left(1-\gamma^{5}\right) h, & & \tilde{T}_{1}^{q}=\bar{h} \gamma_{\mu}\left(1-\gamma^{5}\right) T^{A} q \bar{q} \gamma^{\mu}\left(1-\gamma^{5}\right) T^{A} h, \\
\tilde{Q}_{2}^{q}=\bar{h}\left(1-\gamma^{5}\right) q \bar{q}\left(1+\gamma^{5}\right) h, & & \tilde{T}_{2}^{q}=\bar{h}\left(1-\gamma^{5}\right) T^{A} q \bar{q}\left(1+\gamma^{5}\right) T^{A} h .
\end{array}
$$

Our basis of evanescent operators and the results of the matching computation can be found in appendix A.2. We only consider the isospin-breaking combinations of operators

$$
Q_{i}=Q_{i}^{u}-Q_{i}^{d}, \quad T_{i}=T_{i}^{u}-T_{i}^{d},
$$




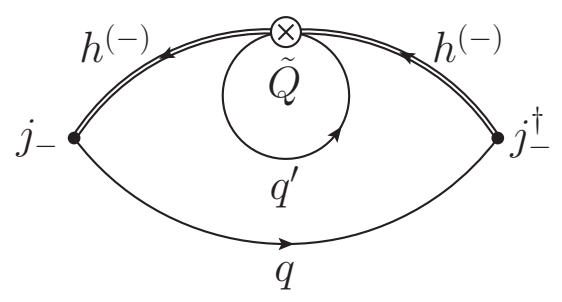

Figure 5. Leading order eye contraction.

and their analogues in HQET. This implies that the eye contractions displayed in figure 5 cancel in the limit of exact isospin symmetry.

The matrix elements are

$$
\left\langle Q_{i}(\mu)\right\rangle=A_{i} f_{B}^{2} M_{B}^{2} B_{i}(\mu), \quad\left\langle T_{i}(\mu)\right\rangle=A_{i} f_{B}^{2} M_{B}^{2} \epsilon_{i}(\mu),
$$

where $\langle Q\rangle=\left\langle B^{-}|Q| B^{-}\right\rangle$, the coefficients read

$$
A_{1}=1, \quad A_{2}=\frac{M_{B}^{2}}{\left(m_{b}+m_{q}\right)^{2}},
$$

and $B_{i}=1, \epsilon_{i}=0$ corresponds to the VSA approximation. Similarly we obtain for the HQET operators

$$
\left\langle\tilde{Q}_{i}(\mu)\right\rangle=\tilde{A}_{i} F^{2}(\mu) \tilde{B}_{i}(\mu), \quad\left\langle\tilde{T}_{i}(\mu)\right\rangle=\tilde{A}_{i} F^{2}(\mu) \tilde{\epsilon}_{i}(\mu),
$$

where

$$
\tilde{A}_{1}=1, \quad \tilde{A}_{2}=1 .
$$

\subsection{Results for the spectral functions and bag parameters}

For the $\Delta B=0$ operators we use the same conventions for the decomposition of the threepoint correlator and the sum rule as for the $\Delta B=2$ operators above. We obtain for the double discontinuities of the non-factorizable contributions

$$
\begin{aligned}
& r_{\tilde{Q}_{i}}\left(x, L_{\omega}\right)=0, \\
& r_{\tilde{T}_{1}}\left(x, L_{\omega}\right)=-8+\frac{a_{1}}{8}+\frac{2 \pi^{2}}{3}-\frac{3}{2} L_{\omega}-\frac{1}{4} \phi(x), \\
& r_{\tilde{T}_{2}}\left(x, L_{\omega}\right)=-\frac{29}{4}+\frac{a_{2}}{8}+\frac{2 \pi^{2}}{3}-\frac{3}{2} L_{\omega}-\frac{1}{4} \phi(x) .
\end{aligned}
$$

The leading condensate contributions have been determined in [14]. From their results we deduce that

$$
\begin{aligned}
& \rho_{\tilde{Q}_{i}}^{\text {cond }}\left(\omega_{1}, \omega_{2}\right)=0+\ldots \\
& \rho_{\tilde{T}_{1}}^{\text {cond }}\left(\omega_{1}, \omega_{2}\right)=\frac{\left\langle g_{s} \bar{q} \sigma_{\mu \nu} G^{\mu \nu} q\right\rangle}{128 \pi^{2}}\left[\delta\left(\omega_{1}\right)+\delta\left(\omega_{2}\right)\right]+\ldots, \\
& \rho_{\tilde{T}_{2}}^{\text {cond }}\left(\omega_{1}, \omega_{2}\right)=-\frac{1}{64 \pi^{2}}\left[\left\langle\frac{\alpha_{s}}{\pi} G^{2}\right\rangle+\left\langle g_{s} \bar{q} \sigma_{\mu \nu} G^{\mu \nu} q\right\rangle\left[\delta\left(\omega_{1}\right)+\delta\left(\omega_{2}\right)\right]\right]+\ldots
\end{aligned}
$$


where the dots indicate factorizable contributions, $\alpha_{s}$ corrections and contributions from condensates of dimension six and higher. To determine the condensate contributions to the HQET parameters we have used the traditional form of the sum rule, because the appearance of the $\delta$-functions obviously prevents the application of a weight function analogous to $(3.24)$. We find

$$
\begin{aligned}
\Delta \tilde{B}_{i}^{\text {cond }}(1.5 \mathrm{GeV}) & =0.000 \pm 0.002 \\
\Delta \tilde{\epsilon}_{1}^{\text {cond }}(1.5 \mathrm{GeV}) & =-0.005 \pm 0.003 \\
\Delta \tilde{\epsilon}_{2}^{\text {cond }}(1.5 \mathrm{GeV}) & =+0.006 \pm 0.004
\end{aligned}
$$

The associated errors were determined from an uncertainty of \pm 0.002 for missing higherdimensional condensates, variations of the Borel parameters and the continuum cutoff and the uncertainty in the condensates

$$
\left\langle\frac{\alpha_{s}}{\pi} G^{2}\right\rangle=(0.012 \pm 0.006) \mathrm{GeV}^{4}, \quad\left\langle g_{s} \bar{q} \sigma_{\mu \nu} G^{\mu \nu} q\right\rangle=(-0.011 \pm 0.002) \mathrm{GeV}^{5} .
$$

We note that our results for the contributions of the condensate corrections to the deviation of the Bag parameters from the VSA are much smaller than those of [14]. This is mostly due to the choice of the Borel parameter. We use $t \sim 1 \mathrm{GeV}$ where the sum rule is stable against variations of the Borel parameter, while the Borel region of [14] translates to $t=(0.35-0.5) \mathrm{GeV}$ where the sum rule becomes unstable as can be seen in their plots. Our choice is also preferred by other modern sum rule analyses [12, 13, 80, 82].

Following analysis strategy for the perturbative contributions described for the $\Delta B=2$ Bag parameters in section 4.1, we find the HQET Bag parameters

$$
\begin{aligned}
\tilde{B}_{1}(1.5 \mathrm{GeV}) & \left.\left.=1.000_{-0.020}^{+0.020}=1.000_{-0.000}^{+0.000}(\bar{\Lambda})_{-0.020}^{+0.020} \text { (intr. }\right)_{-0.002}^{+0.002} \text { (cond. }\right)_{-0.001}^{+0.000}\left(\mu_{\rho}\right), \\
\tilde{B}_{2}(1.5 \mathrm{GeV}) & \left.\left.=1.000_{-0.020}^{+0.020}=1.000_{-0.000}^{+0.000}(\bar{\Lambda})_{-0.020}^{+0.020} \text { (intr. }\right)_{-0.002}^{+0.002} \text { (cond. }\right)_{-0.001}^{+0.000}\left(\mu_{\rho}\right), \\
\tilde{\epsilon}_{1}(1.5 \mathrm{GeV}) & \left.\left.=-0.016_{-0.022}^{+0.021}=-0.016_{-0.008}^{+0.007}(\bar{\Lambda})_{-0.020}^{+0.020} \text { (intr. }\right)_{-0.003}^{+0.003} \text { (cond. }\right)_{-0.003}^{+0.003}\left(\mu_{\rho}\right), \\
\tilde{\epsilon}_{2}(1.5 \mathrm{GeV}) & \left.\left.=0.004_{-0.022}^{+0.022}=0.004_{-0.008}^{+0.007}(\bar{\Lambda})_{-0.020}^{+0.020} \text { (intr. }\right)_{-0.004}^{+0.004} \text { (cond. }\right)_{-0.002}^{+0.002}\left(\mu_{\rho}\right) .
\end{aligned}
$$

where we have set $a_{1}=a_{2}=0$. At the considered order there is no deviation from the VSA for the Bag parameters of the color singlet operators, as can be seen in (5.8) and (5.9), because the corresponding color factors vanish. The deviations for the color octet operators are in the range $0-2 \%$ for scales $\mu_{\rho}$ between 1 and $2 \mathrm{GeV}$. In QCD we obtain

$$
\begin{aligned}
\bar{B}_{1}\left(\mu=\bar{m}_{b}\left(\bar{m}_{b}\right)\right) & \left.=1.028_{-0.056}^{+0.064}=1.028_{-0.019}^{+0.019} \text { (sum rule) }\right)_{-0.053}^{+0.061} \text { (matching) }, \\
\bar{B}_{2}\left(\mu=\bar{m}_{b}\left(\bar{m}_{b}\right)\right) & =0.988_{-0.079}^{+0.087}=0.988_{-0.020}^{+0.020} \text { (sum rule) }{ }_{-0.077}^{+0.085} \text { (matching) }, \\
\bar{\epsilon}_{1}\left(\mu=\bar{m}_{b}\left(\bar{m}_{b}\right)\right) & \left.=-0.107_{-0.029}^{+0.028}=-0.107_{-0.024}^{+0.023} \text { (sum rule }\right)_{-0.017}^{+0.015} \text { (matching) } \\
\bar{\epsilon}_{2}\left(\mu=\bar{m}_{b}\left(\bar{m}_{b}\right)\right) & =-0.033_{-0.021}^{+0.021}=-0.033_{-0.018}^{+0.018} \text { (sum rule) }{ }_{-0.011}^{+0.011} \text { (matching) } .
\end{aligned}
$$

The RG evolution and the perturbative matching cause larger deviations from the VSA which, however, do not exceed $11 \%$. In figure 6 we compare our results to previous ones from sum rules $[14,15]$ and the lattice $[22,23]$. The results of $[14,15,22]$ were obtained within HQET. For the comparison we match their results to QCD at tree level while 


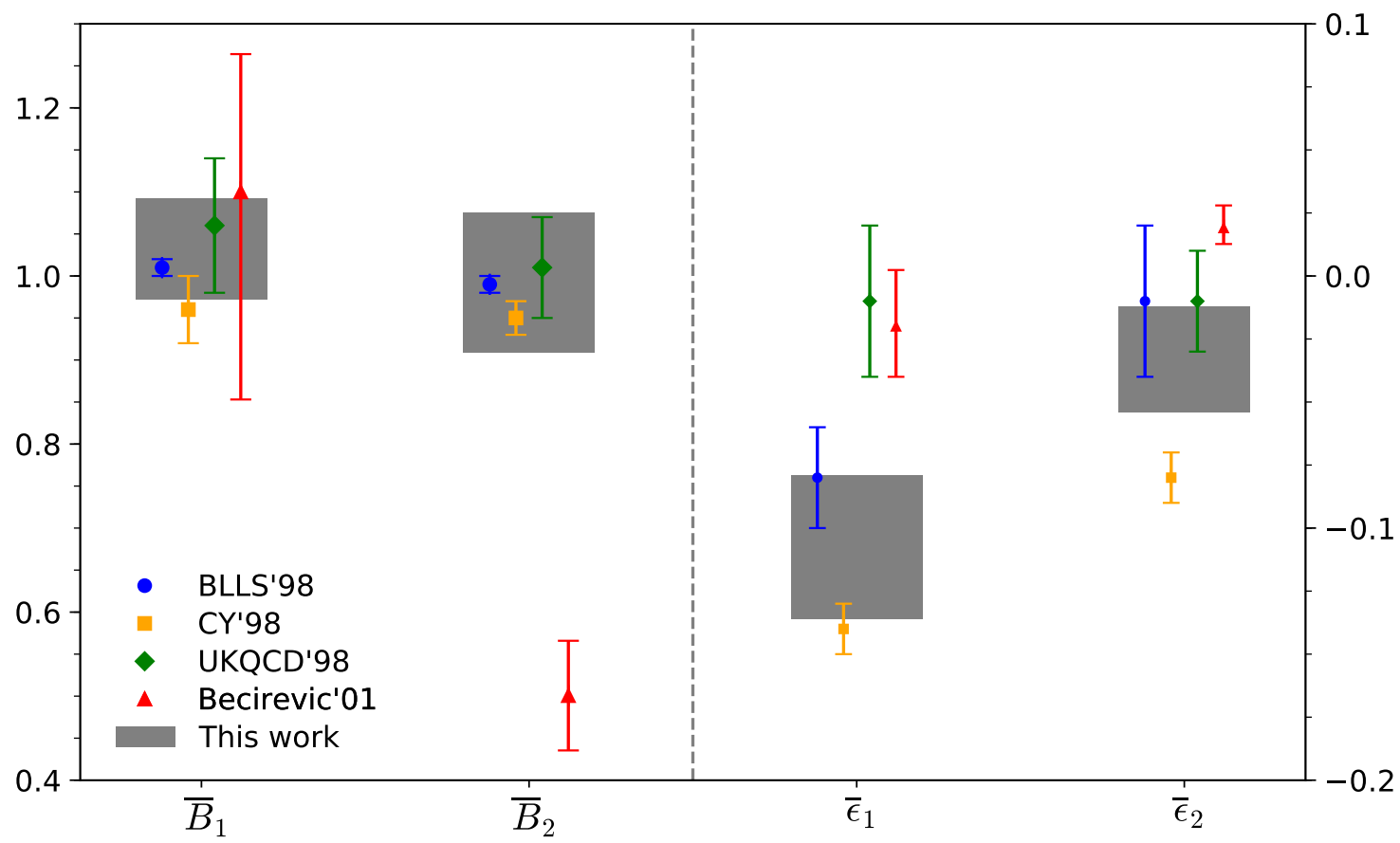

Figure 6. Comparison of our results for the $\Delta B=0$ Bag parameters at the scale $\bar{m}_{b}\left(\bar{m}_{b}\right)$ to the HQET sum rule results BLLS'98 [14] and CY'98 [15], and the lattice values of UKQCD'98 [22] and Becirevic'01 [23].

expanding factors of $\tilde{A}_{i} / \bar{A}_{Q}\left(\bar{m}_{b}\left(\bar{m}_{b}\right)\right)$ in $1 / m_{b}$. As discussed in section 4.1 this effectively includes $1 / m_{b}$ corrections in the VSA approximation.

The $\bar{B}_{i}$ are in good agreement, with the exception of the value for $\bar{B}_{2}$ from [23], which differs from the other results and the VSA by a factor of about two. While the other sum rule results for the $\bar{\epsilon}_{i}$ agree reasonably well with ours, the lattice results for $\bar{\epsilon}_{1}$ show significantly smaller deviations from the VSA. The similarity between the sum rule results $[14,15]$ and ours appears to be mostly coincidental. As discussed above, we find that the bulk of the deviation from the VSA in the $\bar{\epsilon}_{i}$ is due to the RG running and matching, while the latter was not considered in $[14,15]$. In their analyses, there is instead a sizeable deviation at the hadronic scale, originating from the condensate contributions. In comparison with [14] we find that this is due to the choice of very small values of the Borel parameter which lie outside of the stability region as discussed above. The assessment of the origin of the smallness of the lattice results $[22,23]$ for the $\bar{\epsilon}_{i}$ is beyond the scope of this work. Many of the approximations made in [22, 23], like quenching, have since been reappraised and a comparison with a state-of-the art lattice simulation is required. 


\subsection{Results for the lifetime ratios}

Using our results (5.13) for the dimension-six Bag parameters and the VSA for the dimension-seven Bag parameters defined in [29], $\rho_{i}=1 \pm 1 / 12, \sigma_{i}=0 \pm 1 / 6$,

$$
\begin{aligned}
& \left.\frac{\tau\left(B^{+}\right)}{\tau\left(B^{0}\right)}\right|_{\text {exp }}=1.076 \pm 0.004, \\
& \left.\frac{\tau\left(B^{+}\right)}{\tau\left(B^{0}\right)}\right|_{\overline{\mathrm{MS}}}=1.078_{-0.023}^{+0.021} \quad=1.078_{-0.019}^{+0.020} \text { (had.) }{ }_{-0.011}^{+0.002} \text { (scale) } \pm 0.006 \text { (param.), } \\
& \left.\frac{\tau\left(B^{+}\right)}{\tau\left(B^{0}\right)}\right|_{\mathrm{PS}}=1.082_{-0.026}^{+0.022}=1.082 \pm 0.021 \text { (had.) }{ }_{-0.015}^{+0.000} \text { (scale) } \pm 0.006 \text { (param.), } \\
& \left.\frac{\tau\left(B^{+}\right)}{\tau\left(B^{0}\right)}\right|_{1 \mathrm{~S}}=1.082_{-0.028}^{+0.023} \quad=1.082_{-0.021}^{+0.022} \text { (had.) }{ }_{-0.017}^{+0.001}(\text { scale })_{-0.006}^{+0.007} \text { (param.) }, \\
& \left.\left.\frac{\tau\left(B^{+}\right)}{\tau\left(B^{0}\right)}\right|_{\text {kin }}=1.081_{-0.027}^{+0.022} \quad=1.081 \pm 0.021 \text { (had. }\right)_{-0.016}^{+0.001} \text { (scale) } \pm 0.006 \text { (param.), }
\end{aligned}
$$

we find excellent agreement with the experimental value and very good consistency between different mass schemes. The biggest contributions to the total uncertainty are still from the hadronic matrix elements, specifically from $\epsilon_{1}$ with \pm 0.015 and $\sigma_{3}$ with \pm 0.013 . In the future, they can be reduced with an independent determination of the dimension-six Bag parameters and a sum-rule determination of the dimension-seven Bag parameters.

We also update the prediction for the lifetime ratio $\tau\left(B_{s}^{0}\right) / \tau\left(B^{0}\right)$ in the $\overline{\mathrm{MS}}$ scheme using eq. (117) from [16]:

$$
\begin{aligned}
\left.\frac{\tau\left(B_{s}^{0}\right)}{\tau\left(B^{0}\right)}\right|_{\exp } & =0.994 \pm 0.004 \\
\left.\frac{\tau\left(B_{s}^{0}\right)}{\tau\left(B^{0}\right)}\right|_{\overline{\mathrm{MS}}} & =0.9994 \pm 0.0025 \\
& =0.9994 \pm 0.0014 \text { (had.) } \pm 0.0006 \text { (scale) } \pm 0.0020\left(1 / m_{b}^{4}\right),
\end{aligned}
$$

where we have added an uncertainty estimate for the spectator effects at order $1 / m_{b}^{4}$ which have not been considered in [16]. With respect to last year [6], the difference between the theory prediction and the experimental value for $\tau\left(B_{s}^{0}\right) / \tau\left(B^{0}\right)$ is reduced from $2.5 \sigma$ to $1.1 \sigma$.

\section{Matrix elements for charm and the $D^{+}-D^{0}$ lifetime ratio}

The HQET sum rule analysis can easily be adapted to the charm sector. It is common to quote the matrix elements for the charm sector at the scale $3 \mathrm{GeV}$ instead of the charmquark mass, see [30-32], and we adopt that convention for ease of comparison. Consequently we also use $3 \mathrm{GeV}$ as the central matching scale. In the error analysis it is varied between 2 and $4 \mathrm{GeV}$. To account for the lower value of charm-quark mass we assume that the uncertainty due to power corrections is 0.03 instead of 0.01 for the bottom sector. Otherwise we use the same analysis strategy as in the bottom sector which is outlined in section 4.1.

\subsection{Matrix elements for $D$ mixing}

The latest lattice QCD study [32] for $D$ mixing only gives results for the matrix elements and not for the Bag parameters. We do the same here and obtain, using the value of the 


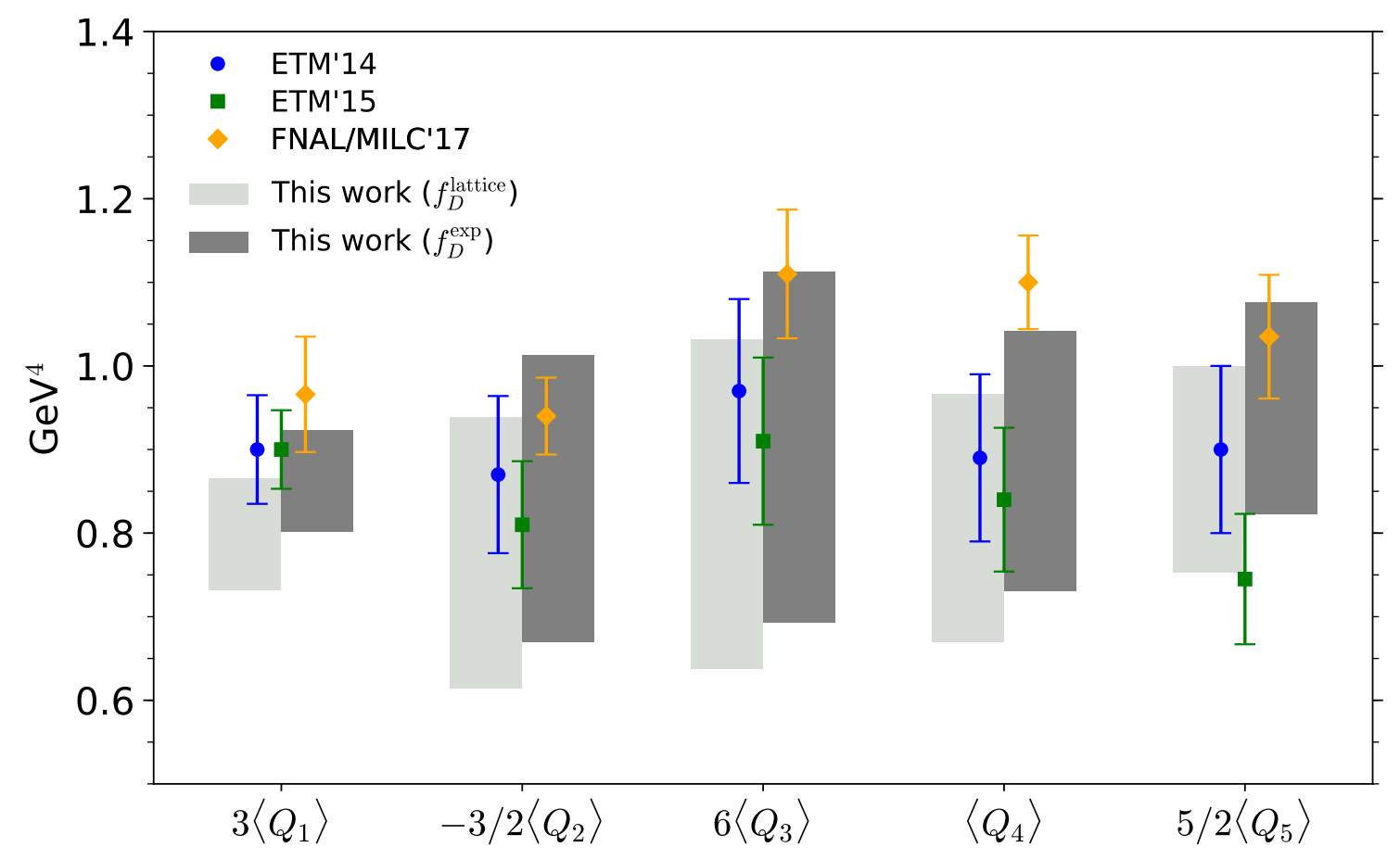

Figure 7. Comparison of our results for the $\Delta C=2$ matrix elements at the scale $3 \mathrm{GeV}$ to the lattice values of ETM'14 [30], ETM'15 [31] and FNAL/MILC'17 [32]. The values for the matrix elements of the ETM collaboration are extracted from figure 16 of [32].

$D$-meson decay constant from appendix B,

$$
\begin{aligned}
\left\langle Q_{1}(3 \mathrm{GeV})\right\rangle / \mathrm{GeV}^{4} & \left.\left.=0.265_{-0.021}^{+0.024}=0.265_{-0.010}^{+0.006} \text { (s.r. }\right)_{-0.014}^{+0.019} \text { (matching }\right)_{-0.012}^{+0.013}\left(f_{D}\right) \\
-\left\langle Q_{2}(3 \mathrm{GeV})\right\rangle / \mathrm{GeV}^{4} & \left.\left.=0.502_{-0.092}^{+0.124}=0.502_{-0.078}^{+0.094} \text { (s.r. }\right)_{-0.044}^{+0.076} \text { (matching }\right)_{-0.023}^{+0.024}\left(f_{D}\right) \\
\left\langle Q_{3}(3 \mathrm{GeV})\right\rangle / \mathrm{GeV}^{4} & \left.\left.=0.135_{-0.029}^{+0.037}=0.135_{-0.026}^{+0.031} \text { (s.r. }\right)_{-0.010}^{+0.019} \text { (matching) }\right)_{-0.006}^{+0.006}\left(f_{D}\right) \\
\left\langle Q_{4}(3 \mathrm{GeV})\right\rangle / \mathrm{GeV}^{4} & \left.\left.=0.792_{-0.122}^{+0.175}=0.792_{-0.093}^{+0.116} \text { (s.r. }\right)_{-0.070}^{+0.125} \text { (matching) }\right)_{-0.037}^{+0.038}\left(f_{D}\right) \\
\left\langle Q_{5}(3 \mathrm{GeV})\right\rangle / \mathrm{GeV}^{4}=0.340_{-0.039}^{+0.060} & \left.\left.=0.340_{-0.021}^{+0.027} \text { (s.r. }\right)_{-0.029}^{+0.051} \text { (matching) }\right)_{-0.016}^{+0.016}\left(f_{D}\right)
\end{aligned}
$$

The relative uncertainties in the charm sector are consistently larger than those in the bottom sector because of larger perturbative corrections due to a larger value of $\alpha_{s}$ at the smaller scales and larger power corrections. This effect is most pronounced for $Q_{2}, Q_{4}$ and $Q_{5}$ where the relative uncertainty is larger by a factor of order two. In the matrix elements we have an additional uncertainty from the value of the decay constant which is added in quadrature.

We compare our results to those from the lattice in figure 7 . There is a consistent hierarchy with decreasing values from the results of the FNAL/MILC collaboration [32], those of the ETM collaboration [30,31] and ours. The only exception is the value of $\left\langle Q_{5}\right\rangle$ from [31] which lies below ours. If we use the lattice average [60] for the decay constant $f_{D}^{\text {lattice }}=(211.9 \pm 1.1) \mathrm{MeV}$ in place of the experimental average $f_{D}^{\exp }=(203.7 \pm$ 4.8) $\mathrm{MeV}$ [60], we find very good agreement between our results and those of ETM and the 
remaining differences with respect to the FNAL/MILC results are comfortably below two sigmas. We prefer the experimental average of the decay constant since it is in significantly better agreement with recent sum rule results [79-82]. On the other hand, using the lattice value yields a more meaningful comparison with the lattice results since the quantities we determine with the sum rule are the Bag parameters and the decay constant cancels out in the comparison if the same value is used on both sides. We therefore conclude that our sum rule results for the non-factorizable contributions to the Bag parameters are in good agreement with lattice simulations. An investigation of the differences in the numerical values of the decay constant is beyond the scope of this work.

\subsection{Matrix elements for $D$ lifetimes and $\tau\left(D^{+}\right) / \tau\left(D^{0}\right)$}

Our results for the $\Delta C=0$ Bag parameters are

$$
\begin{aligned}
\bar{B}_{1}(3 \mathrm{GeV}) & =0.902_{-0.051}^{+0.077}=0.902_{-0.018}^{+0.018} \text { (sum rule) }{ }_{-0.048}^{+0.075} \text { (matching), } \\
\bar{B}_{2}(3 \mathrm{GeV}) & \left.=0.739_{-0.073}^{+0.124}=0.739_{-0.015}^{+0.015} \text { (sum rule }\right)_{-0.072}^{+0.123} \text { (matching), } \\
\bar{\epsilon}_{1}(3 \mathrm{GeV}) & =-0.132_{-0.046}^{+0.041}=-0.132_{-0.026}^{+0.025} \text { (sum rule) }{ }_{-0.038}^{+0.033} \text { (matching) }, \\
\bar{\epsilon}_{2}(3 \mathrm{GeV}) & =-0.005_{-0.032}^{+0.032}=-0.005_{-0.012}^{+0.011} \text { (sum rule) }{ }_{-0.030}^{+0.030} \text { (matching). }
\end{aligned}
$$

While the uncertainties in $B_{1,2}$ are similar to those in the $B$ sector we find that those in $\epsilon_{1,2}$ are larger by about $50 \%$. The latter ones are dominated by the non-factorizable power correction and the intrinsic sum rule errors which are both based on somewhat ad-hoc estimates. Thus, our values for the uncertainties of $\epsilon_{1,2}$ should be taken with a grain of salt and lattice results for the $\Delta C=0$ Bag parameters could provide an important consistency check. Alternatively, one could also improve the dominant error due to non-factorizable $1 / m_{c}$ corrections by performing the operator matching up to the order $1 / m_{c}$ and determine the matrix elements of the subleading HQET operators using sum rules.

We update our result for the $D$-meson lifetime ratio from [29] using the dimension six Bag parameters (6.2) and the VSA $\rho_{i}=1 \pm 1 / 12, \sigma_{i}=0 \pm 1 / 6$ for the dimension-seven Bag parameters. We have converted the $\overline{\mathrm{MS}}$ value of the charm-quark mass to the PS mass at $\mu_{f}=1 \mathrm{GeV}$ and the $1 \mathrm{~S}$ mass at four-loop accuracy using RunDec. The kinetic mass at the scale $1 \mathrm{GeV}$ is determined with two-loop accuracy using an unpublished version of the QQbar_Threshold code [83, 84]. The central value for the scales $\mu_{1}$ and $\mu_{0}$ is fixed to $1.5 \mathrm{GeV}$ for all mass schemes and varied between 1 and $3 \mathrm{GeV}$. We find

$$
\begin{aligned}
& \left.\frac{\tau\left(D^{+}\right)}{\tau\left(D^{0}\right)}\right|_{\exp }=2.536 \pm 0.019 \\
& \left.\frac{\tau\left(D^{+}\right)}{\tau\left(D^{0}\right)}\right|_{\overline{\mathrm{MS}}}=2.61_{-0.77}^{+0.72}=2.61_{-0.66}^{+0.70} \text { (had.) }{ }_{-0.38}^{+0.12} \text { (scale) } \pm 0.09 \text { (param.), } \\
& \left.\frac{\tau\left(D^{+}\right)}{\tau\left(D^{0}\right)}\right|_{\mathrm{PS}}=2.70_{-0.82}^{+0.74}=2.70_{-0.68}^{+0.72} \text { (had.) }{ }_{-0.45}^{+0.11} \text { (scale) } \pm 0.10 \text { (param.), } \\
& \left.\frac{\tau\left(D^{+}\right)}{\tau\left(D^{0}\right)}\right|_{1 \mathrm{~S}}=2.56_{-0.99}^{+0.81}=2.56_{-0.74}^{+0.78} \text { (had.) }{ }_{-0.65}^{+0.22} \text { (scale) } \pm 0.10 \text { (param.), } \\
& \left.\frac{\tau\left(D^{+}\right)}{\tau\left(D^{0}\right)}\right|_{\text {kin }}=2.53_{-0.76}^{+0.72}=2.53_{-0.66}^{+0.70} \text { (had.) }{ }_{-0.37}^{+0.13} \text { (scale) } \pm 0.10 \text { (param.), }
\end{aligned}
$$


which is in very good agreement. The various mass schemes are all consistent and we again take the PS result as our preferred value. The dominant sources of uncertainties are the Bag parameters $\epsilon_{1}$ and $\sigma_{3}$ which both contribute \pm 0.5 to the error budget of the lifetime ratio. Both errors can be reduced in the future with a lattice determination of the dimensions-six matrix elements and a sum-rule determination of the dimension-seven Bag parameters, respectively. In the PS scheme, the radiative and power corrections are of the order $+27 \%$ and $-34 \%$, respectively, which indicates good convergence behaviour. We therefore conclude that the HQE provides a good description of the lifetime ratio $\tau\left(D^{+}\right) / \tau\left(D^{0}\right)$.

\section{Conclusions}

We have determined the matrix elements of the dimension six $\Delta F=0,2$ operators for the bottom and charm sector using HQET sum rules. Our findings for the $\Delta F=2$ matrix elements are in good agreement with recent lattice [3-5, 30-32] and sum rule [11] results. Our $\Delta F=0$ results are the first state-of-the-art values for the matrix elements required for $B$ and $D$ meson lifetime ratios. The uncertainties in our analyses for the Bag parameters are similar to those of recent lattice determinations in the $B$ sector and somewhat larger in the $D$ sector. This suggests that the uncertainty of the $\Delta C=0$ matrix elements could be reduced by a lattice simulation. In most cases, the dominant errors in our approach stem from the matching of QCD to HQET operators, see appendix B. These could be reduced substantially by performing the matching calculation at NNLO. Some first steps towards this goal have recently been taken in [70]. Consequently, in the future, sum rules will continue to be competitive with lattice simulations in the determination of four-quark operators.

Our predictions for the mixing observables and lifetime ratios in the $B$ sector are in good agreement with the experimental averages as summarized in figures 8 and 9 . In particular, the small tensions [5, 6] that follow from using the FNAL/MILC results [5] for the matrix elements are not confirmed by our results. We note that the predictions based on matrix elements from sum rules and from lattice simulations are compatible and lead to overall uncertainties of the same size. Taking the naive average of the Bag parameters, the relative uncertainties of the mass and decay rate difference are, however, only reduced by about $9 \%$ and $6 \%$, respectively, because other sources of uncertainties, like e.g. the matrix elements of dimension-seven operators, are dominant.

We find that the experimental value for the lifetime ratio $\tau\left(D^{+}\right) / \tau\left(D^{0}\right)$ can be reproduced within the HQE. This is a strong indication that the HQE does not break down in the charm sector. However, due to sizeable hadronic uncertainties, we cannot exclude large duality violations at the level of $20-30 \%$ yet. On the other hand, the $D$-mixing observables are very sensitive to duality violations and might offer a handle on a better quantitative understanding of these effects [25].

Our comprehensive study demonstrates that the HQET sum rules for hadronic fourquark matrix elements provide a competitive alternative to lattice simulations. Due to completely different systematics they facilitate powerful independent checks of lattice re- 

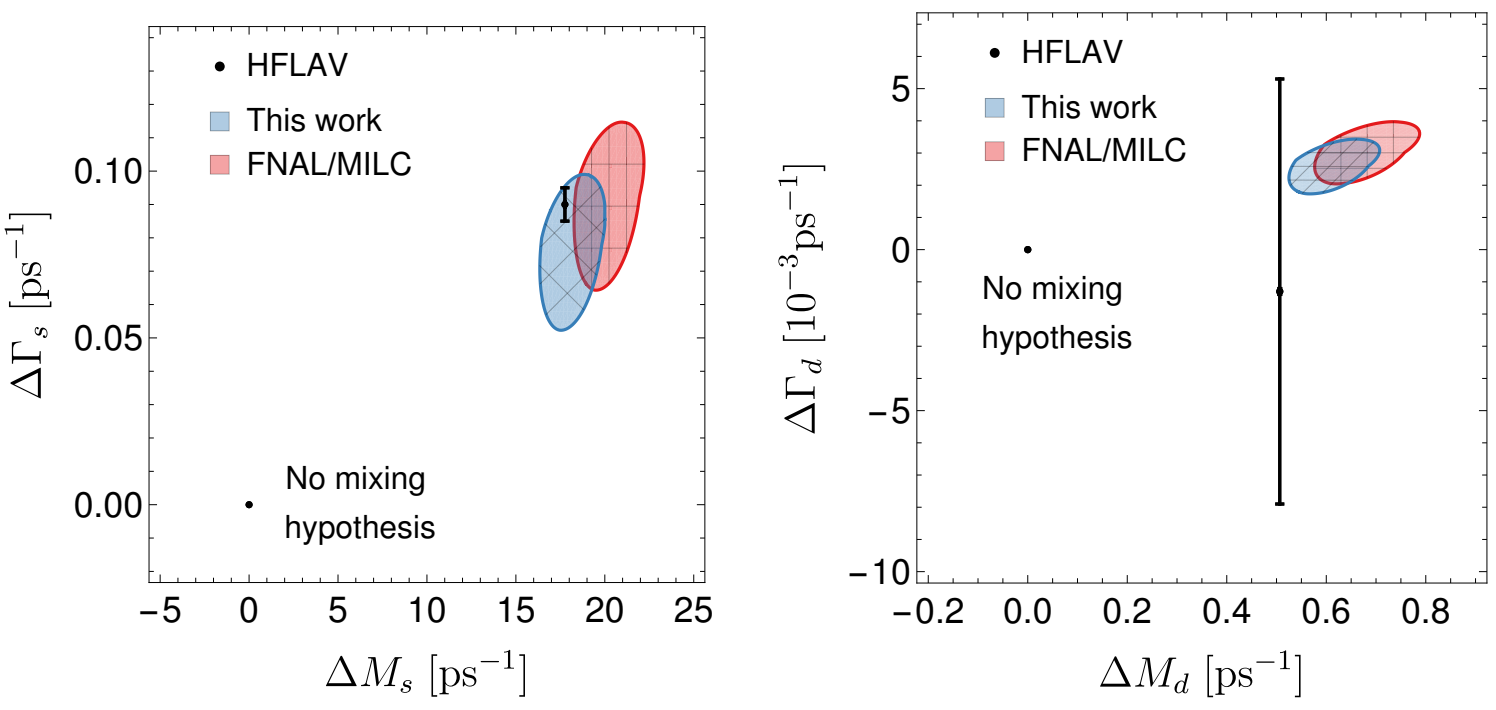

Figure 8. Comparison of our predictions for the mass and decay rate difference in the $B_{s}$ (left) and $B_{d}$ (right) system with the present experimental averages (error bars). We also show the results obtained with the lattice results of [5] for $f_{B_{q}}^{2} \bar{B}_{Q_{i}}$ and the matrix element $\left\langle R_{0}\right\rangle$ and the values given in appendix B for the other input parameters. The PS mass scheme for the bottom quarks has been used in both cases.

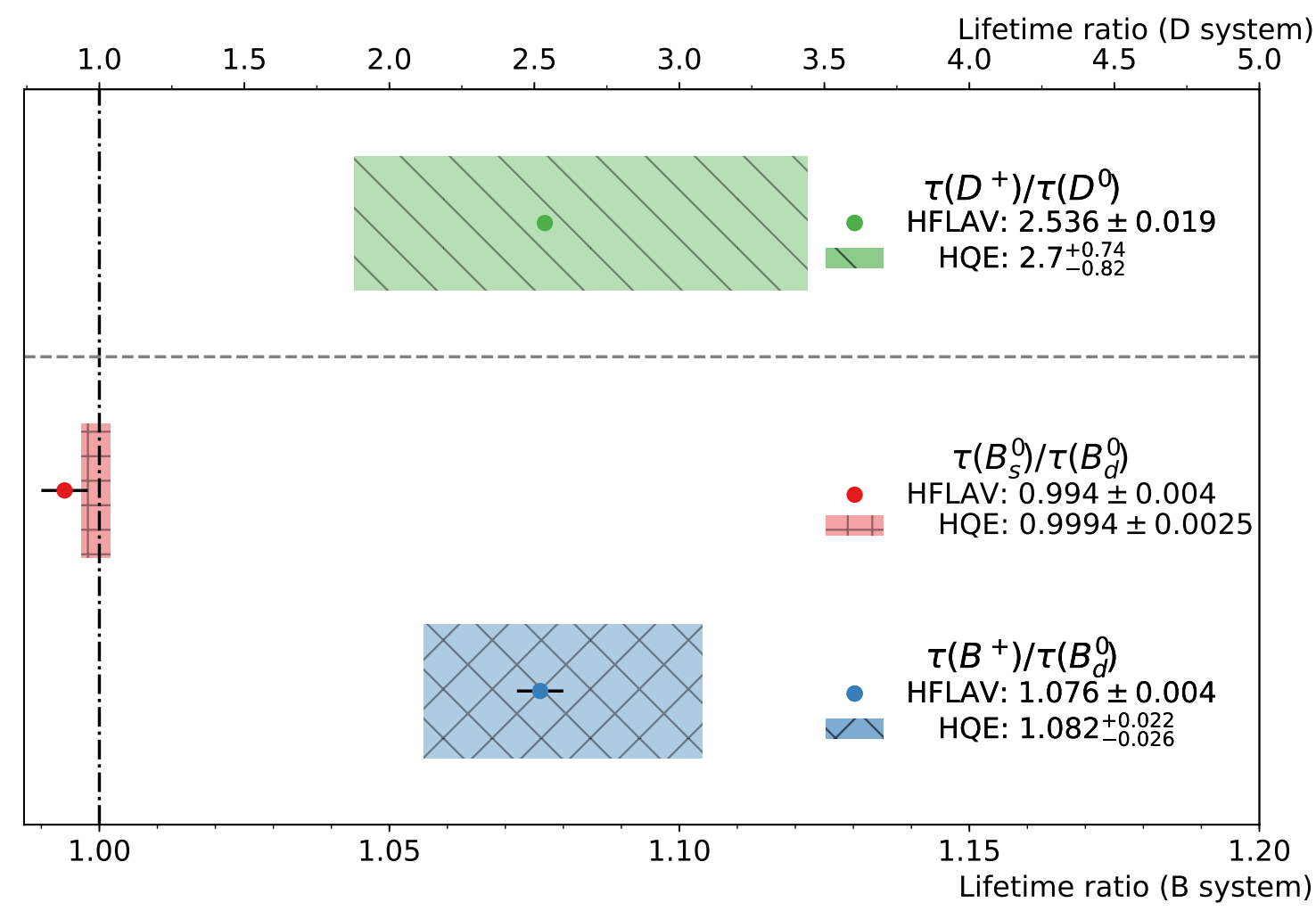

Figure 9. Comparison of our predictions for the lifetime ratios of heavy mesons with the present experimental averages. 
sults. Sum rules can also be applied to obtain the matrix elements of the subleading dimension-seven operators, which have never been determined using lattice simulations. This is crucial to achieve a substantial reduction of the current theoretical uncertainties.

\section{Acknowledgments}

We are grateful to V. Braun for illuminating discussions. This work was supported by the STFC through the IPPP grant.

\section{A Basis of evanescent operators and ADMs}

\section{A.1 $\Delta B=2$ operators}

Our choice of basis for the evanescent operators is given by

$$
\begin{aligned}
& E_{1}=\bar{b}_{i} \gamma_{\mu}\left(1-\gamma^{5}\right) q_{j} \bar{b}_{j} \gamma^{\mu}\left(1-\gamma^{5}\right) q_{i}-Q_{1}, \\
& E_{2}=\bar{b}_{i} \gamma_{\mu} \gamma_{\nu}\left(1-\gamma^{5}\right) q_{i} \bar{b}_{j} \gamma^{\mu} \gamma^{\nu}\left(1-\gamma^{5}\right) q_{j}-(8-4 \epsilon) Q_{2}-(8-8 \epsilon) Q_{3}, \\
& E_{3}=\bar{b}_{i} \gamma_{\mu} \gamma_{\nu}\left(1-\gamma^{5}\right) q_{j} \bar{b}_{j} \gamma^{\mu} \gamma^{\nu}\left(1-\gamma^{5}\right) q_{i}-(8-8 \epsilon) Q_{2}-(8-4 \epsilon) Q_{3}, \\
& E_{4}=\bar{b}_{i} \gamma_{\mu} \gamma_{\nu} \gamma_{\rho}\left(1-\gamma^{5}\right) q_{i} \bar{b}_{j} \gamma^{\mu} \gamma^{\nu} \gamma^{\rho}\left(1-\gamma^{5}\right) q_{j}-(16-4 \epsilon) Q_{1}, \\
& E_{5}=\bar{b}_{i} \gamma_{\mu} \gamma_{\nu} \gamma_{\rho}\left(1-\gamma^{5}\right) q_{j} \bar{b}_{j} \gamma^{\mu} \gamma^{\nu} \gamma^{\rho}\left(1-\gamma^{5}\right) q_{i}-(16-4 \epsilon)\left(Q_{1}+E_{1}\right), \\
& E_{6}=\bar{b}_{i} \gamma_{\mu}\left(1-\gamma^{5}\right) q_{i} \bar{b}_{j} \gamma^{\mu}\left(1+\gamma^{5}\right) q_{j}+2 Q_{5}, \\
& E_{7}=\bar{b}_{i} \gamma_{\mu}\left(1-\gamma^{5}\right) q_{j} \bar{b}_{j} \gamma^{\mu}\left(1+\gamma^{5}\right) q_{i}+2 Q_{4}, \\
& E_{8}=\bar{b}_{i} \gamma_{\mu} \gamma_{\nu}\left(1-\gamma^{5}\right) q_{i} \bar{b}_{j} \gamma^{\mu} \gamma^{\nu}\left(1+\gamma^{5}\right) q_{j}-4 Q_{4}, \\
& E_{9}=\bar{b}_{i} \gamma_{\mu} \gamma_{\nu}\left(1-\gamma^{5}\right) q_{j} \bar{b}_{j} \gamma^{\mu} \gamma^{\nu}\left(1+\gamma^{5}\right) q_{i}-4 Q_{5},
\end{aligned}
$$

for QCD and

$$
\begin{aligned}
& \tilde{E}_{1}=\bar{h}_{i}^{\{(+)} \gamma_{\mu}\left(1-\gamma^{5}\right) q_{j} \bar{h}_{j}^{(-)\}} \gamma^{\mu}\left(1-\gamma^{5}\right) q_{i}-\tilde{Q}_{1} \\
& \tilde{E}_{2}=\frac{1}{2} \tilde{Q}_{1}+\tilde{Q}_{2}+\bar{h}_{i}^{\{(+)}\left(1-\gamma^{5}\right) q_{j} \bar{h}_{j}^{(-)\}}\left(1-\gamma^{5}\right) q_{i} \\
& \tilde{E}_{3}=\bar{h}_{i}^{\{(+)} \gamma_{\mu} \gamma_{\nu}\left(1-\gamma^{5}\right) q_{i} \bar{h}_{j}^{(-)\}} \gamma^{\mu} \gamma^{\nu}\left(1-\gamma^{5}\right) q_{j}+\left(4+a_{1} \epsilon\right) \tilde{Q}_{1}, \\
& \tilde{E}_{4}=\bar{h}_{i}^{\{(+)} \gamma_{\mu} \gamma_{\nu}\left(1-\gamma^{5}\right) q_{j} \bar{h}_{j}^{(-)\}} \gamma^{\mu} \gamma^{\nu}\left(1-\gamma^{5}\right) q_{i}+\left(4+a_{1} \epsilon\right)\left(\tilde{Q}_{1}+\tilde{E}_{1}\right), \\
& \tilde{E}_{5}=\bar{h}_{i}^{\{(+)} \gamma_{\mu} \gamma_{\nu} \gamma_{\rho}\left(1-\gamma^{5}\right) q_{i} \bar{h}_{j}^{(-)\}} \gamma^{\mu} \gamma^{\nu} \gamma^{\rho}\left(1-\gamma^{5}\right) q_{j}-\left(16+a_{2} \epsilon\right) \tilde{Q}_{1}, \\
& \tilde{E}_{6}=\bar{h}_{i}^{\{(+)} \gamma_{\mu} \gamma_{\nu} \gamma_{\rho}\left(1-\gamma^{5}\right) q_{j} \bar{h}_{j}^{(-)\}} \gamma^{\mu} \gamma^{\nu} \gamma^{\rho}\left(1-\gamma^{5}\right) q_{i}-\left(16+a_{2} \epsilon\right)\left(\tilde{Q}_{1}+\tilde{E}_{1}\right), \\
& \tilde{E}_{7}=\bar{h}_{i}^{\{(+)} \gamma_{\mu}\left(1-\gamma^{5}\right) q_{i} \bar{h}_{j}^{(-)\}} \gamma^{\mu}\left(1+\gamma^{5}\right) q_{j}+2 \tilde{Q}_{5}, \\
& \tilde{E}_{8}=\bar{h}_{i}^{\{(+)} \gamma_{\mu}\left(1-\gamma^{5}\right) q_{j} \bar{h}_{j}^{(-)\}} \gamma^{\mu}\left(1+\gamma^{5}\right) q_{i}+2 \tilde{Q}_{4}, \\
& \tilde{E}_{9}=\bar{h}_{i}^{\{(+)} \gamma_{\mu} \gamma_{\nu}\left(1-\gamma^{5}\right) q_{i} \bar{h}_{j}^{(-)\}} \gamma^{\mu} \gamma^{\nu}\left(1+\gamma^{5}\right) q_{j}-\left(4+a_{3} \epsilon\right) \tilde{Q}_{4}, \\
& \tilde{E}_{10}=\bar{h}_{i}^{\{(+)} \gamma_{\mu} \gamma_{\nu}\left(1-\gamma^{5}\right) q_{j} \bar{h}_{j}^{(-)\}} \gamma^{\mu} \gamma^{\nu}\left(1+\gamma^{5}\right) q_{i}-\left(4+a_{3} \epsilon\right) \tilde{Q}_{5},
\end{aligned}
$$


for HQET. It is straightforward to verify that the evanescent operators vanish in four dimensions by using the Fierz identities

$$
\begin{aligned}
{\left[\gamma_{\mu}\left(1 \pm \gamma_{5}\right)\right]_{i j}\left[\gamma^{\mu}\left(1 \pm \gamma_{5}\right)\right]_{k l} } & =-\left[\gamma_{\mu}\left(1 \pm \gamma_{5}\right)\right]_{i l}\left[\gamma^{\mu}\left(1 \pm \gamma_{5}\right)\right]_{k j} \\
{\left[1 \pm \gamma_{5}\right]_{i j}\left[1 \pm \gamma_{5}\right]_{k l} } & =\frac{1}{2}\left[1 \pm \gamma_{5}\right]_{i l}\left[1 \pm \gamma_{5}\right]_{k j}+\frac{1}{8}\left[\sigma_{\mu \nu}\left(1 \pm \gamma_{5}\right)\right]_{i l}\left[\sigma^{\mu \nu}\left(1 \pm \gamma_{5}\right)\right]_{k j} \\
{\left[\gamma_{\mu}\left(1 \pm \gamma_{5}\right)\right]_{i j}\left[\gamma^{\mu}\left(1 \mp \gamma_{5}\right)\right]_{k l} } & =2\left[1 \mp \gamma_{5}\right]_{i l}\left[1 \pm \gamma_{5}\right]_{k j}
\end{aligned}
$$

and the relation

$$
\gamma_{\mu} \gamma_{\nu} \gamma_{\rho}=g_{\mu \nu} \gamma_{\rho}+g_{\nu \rho} \gamma_{\mu}-g_{\mu \rho} \gamma_{\nu}-i \epsilon_{\mu \nu \rho \lambda} \gamma^{\lambda} \gamma^{5}
$$

A useful strategy to simplify expressions with two Dirac matrices is to use projection identities, e.g.

$$
\bar{h}^{( \pm)} \gamma_{\mu} \gamma_{\nu}\left(1-\gamma^{5}\right) q= \pm \bar{h}^{( \pm)} \psi \gamma_{\mu} \gamma_{\nu}\left(1-\gamma^{5}\right) q
$$

and then reduce the number of Dirac matrices with eq. (A.4).

In the decomposition (2.8) the LO QCD ADM is

$$
\begin{aligned}
\gamma_{Q Q}^{(0)} & =\left(\begin{array}{ccccccc}
\frac{6\left(N_{c}-1\right)}{N_{c}} & 0 & 0 & 0 & 0 \\
0 & -\frac{2\left(3 N_{c}^{2}-4 N_{c}-1\right)}{N_{c}} & \frac{4 N_{c}-8}{N_{c}} & 0 & 0 \\
0 & \frac{4\left(N_{c}-2\right)\left(N_{c}+1\right)}{N_{c}} & \frac{2\left(N_{c}+1\right)^{2}}{N_{c}} & 0 & 0 \\
0 & 0 & & 0 & -\frac{6\left(N_{c}^{2}-1\right)}{N_{c}} & 0 \\
0 & 0 & 0 & 0 & -6 & \frac{6}{N_{c}}
\end{array}\right), \\
\gamma_{Q E}^{(0)}= & \left(\begin{array}{cccccccccc}
6 & 0 & 0 & -\frac{1}{N_{c}} & 1 & 0 & 0 & 0 & 0 \\
0 & -\frac{1}{N_{c}} & 1 & 0 & 0 & 0 & 0 & 0 & 0 \\
0 & \frac{1}{2} & \frac{N_{c}}{2}-\frac{1}{N_{c}} & 0 & 0 & 0 & 0 & 0 & 0 \\
0 & 0 & 0 & 0 & 0 & 0 & 0 & -\frac{1}{N_{c}} & 1 \\
0 & 0 & 0 & 0 & 0 & 0 & 0 & \frac{1}{2} & \frac{N_{c}}{2}-\frac{1}{N_{c}}
\end{array}\right)
\end{aligned}
$$

In HQET we find

$$
\begin{aligned}
& \tilde{\gamma}_{\tilde{Q} \tilde{Q}}^{(0)}=\left(\begin{array}{ccccccc}
\frac{3}{N_{c}}-3 N_{c} & 0 & & 0 & 0 \\
1+\frac{1}{N_{c}} & -3 N_{c}+4+\frac{7}{N_{c}} & 0 & 0 \\
0 & 0 & & \frac{6}{N_{c}}-3 N_{c} & -3 \\
0 & & 0 & & -3 & \frac{6}{N_{c}}-3 N_{c}
\end{array}\right), \\
& \tilde{\gamma}_{\tilde{Q} \tilde{E}}^{(0)}=\left(\begin{array}{ccccccccccc}
0 & 0 & 0 & 0 & -\frac{1}{4 N_{c}} & \frac{1}{4} & 0 & 0 & 0 & 0 \\
-1 & -4 & -\frac{1}{4 N_{c}} & \frac{1}{4} & 0 & 0 & 0 & 0 & 0 & 0 \\
0 & 0 & 0 & 0 & 0 & 0 & 0 & 0 & -\frac{1}{4 N_{c}} & \frac{1}{4} \\
0 & 0 & 0 & 0 & 0 & 0 & 0 & 0 & \frac{1}{4} & -\frac{1}{4 N_{c}}
\end{array}\right)
\end{aligned}
$$

Our result (A.6) with $N_{c}=3$ differs from the results of $[85,86]$ because we have only used the replacements implied by the basis of evanescent operators (A.1) to simplify products of Dirac matrices. We can reproduce their result by applying 4-dimensional Fierz identities that relate $Q_{1}, Q_{2}$ and $Q_{3}$. The upper left $2 \times 2$ submatrix of (A.8) agrees with [34]. 


\section{A.2 $\Delta B=0$ operators}

We define the basis of evanescent operators in QCD following [75]:

$$
\begin{aligned}
& E_{1}^{q}=\bar{b} \gamma_{\mu} \gamma_{\nu} \gamma_{\rho}\left(1-\gamma^{5}\right) q \bar{q} \gamma^{\rho} \gamma^{\nu} \gamma^{\mu}\left(1-\gamma^{5}\right) b-(4-8 \epsilon) Q_{1}^{q}, \\
& E_{2}^{q}=\bar{b} \gamma_{\mu} \gamma_{\nu}\left(1-\gamma^{5}\right) q \bar{q} \gamma^{\nu} \gamma^{\mu}\left(1+\gamma^{5}\right) b-(4-8 \epsilon) Q_{2}^{q}, \\
& E_{3}^{q}=\bar{b} \gamma_{\mu} \gamma_{\nu} \gamma_{\rho}\left(1-\gamma^{5}\right) T^{A} q \bar{q} \gamma^{\rho} \gamma^{\nu} \gamma^{\mu}\left(1-\gamma^{5}\right) T^{A} b-(4-8 \epsilon) T_{1}^{q}, \\
& E_{4}^{q}=\bar{b} \gamma_{\mu} \gamma_{\nu}\left(1-\gamma^{5}\right) T^{A} q \bar{q} \gamma^{\nu} \gamma^{\mu}\left(1+\gamma^{5}\right) T^{A} b-(4-8 \epsilon) T_{2}^{q} .
\end{aligned}
$$

In HQET we again introduce parameters $a_{1,2}$ to keep track of the scheme dependence

$$
\begin{aligned}
& \tilde{E}_{1}^{q}=\bar{h} \gamma_{\mu} \gamma_{\nu} \gamma_{\rho}\left(1-\gamma^{5}\right) q \bar{q} \gamma^{\rho} \gamma^{\nu} \gamma^{\mu}\left(1-\gamma^{5}\right) h-\left(4+a_{1} \epsilon\right) \tilde{Q}_{1}^{q}, \\
& \tilde{E}_{2}^{q}=\bar{h} \gamma_{\mu} \gamma_{\nu}\left(1-\gamma^{5}\right) q \bar{q} \gamma^{\nu} \gamma^{\mu}\left(1+\gamma^{5}\right) h-\left(4+a_{2} \epsilon\right) \tilde{Q}_{2}^{q}, \\
& \tilde{E}_{3}^{q}=\bar{h} \gamma_{\mu} \gamma_{\nu} \gamma_{\rho}\left(1-\gamma^{5}\right) T^{A} q \bar{q} \gamma^{\rho} \gamma^{\nu} \gamma^{\mu}\left(1-\gamma^{5}\right) T^{A} h-\left(4+a_{1} \epsilon\right) \tilde{T}_{1}^{q}, \\
& \tilde{E}_{4}^{q}=\bar{h} \gamma_{\mu} \gamma_{\nu}\left(1-\gamma^{5}\right) T^{A} q \bar{q} \gamma^{\nu} \gamma^{\mu}\left(1+\gamma^{5}\right) T^{A} h-\left(4+a_{2} \epsilon\right) \tilde{T}_{2}^{q} .
\end{aligned}
$$

The isospin breaking combinations of the evanescent operators are defined in analogy to (5.3). The LO ADM in QCD takes the form

$$
\begin{aligned}
\gamma_{Q Q}^{(0)} & =\left(\begin{array}{cccc}
0 & 0 & 12 & 0 \\
0 & \frac{6}{N_{c}}-6 N_{c} & 0 & 0 \\
3-\frac{3}{N_{c}^{2}} & 0 & -\frac{12}{N_{c}} & 0 \\
0 & 0 & 0 & \frac{6}{N_{c}}
\end{array}\right), \\
\gamma_{Q E}^{(0)} & =\left(\begin{array}{cccc}
0 & 0 & -2 & 0 \\
0 & 0 & 0 & -2 \\
\frac{1}{2 N_{c}^{2}}-\frac{1}{2} & 0 & \frac{2}{N_{c}}-\frac{N_{c}}{2} & 0 \\
0 & \frac{1}{2 N_{c}^{2}}-\frac{1}{2} & 0 & \frac{2}{N_{c}}-\frac{N_{c}}{2}
\end{array}\right) .
\end{aligned}
$$

The HQET result is given by

$$
\begin{aligned}
\tilde{\gamma}_{\tilde{Q} \tilde{Q}}^{(0)} & =\left(\begin{array}{cccc}
\frac{3}{N_{c}}-3 N_{c} & 0 & 6 & 0 \\
0 & \frac{3}{N_{c}}-3 N_{c} & 0 & 6 \\
\frac{3}{2}-\frac{3}{2 N_{c}^{2}} & 0 & -\frac{3}{N_{c}} & 0 \\
0 & \frac{3}{2}-\frac{3}{2 N_{c}^{2}} & 0 & -\frac{3}{N_{c}}
\end{array}\right), \\
\tilde{\gamma}_{\tilde{Q} \tilde{E}}^{(0)} & =\left(\begin{array}{cccc}
0 & 0 & -\frac{1}{2} & 0 \\
0 & 0 & 0 & -\frac{1}{2} \\
\frac{1}{8 N_{c}^{2}}-\frac{1}{8} & 0 & \frac{1}{2 N_{c}}-\frac{N_{c}}{4} & 0 \\
0 & \frac{1}{8 N_{c}^{2}}-\frac{1}{8} & 0 & \frac{1}{2 N_{c}}-\frac{N_{c}}{4}
\end{array}\right) .
\end{aligned}
$$

Our result (A.12) is in agreement with [87, 88] and (A.14) reproduces the result of [89]. ${ }^{4}$ The results (A.13) and (A.15) are new. The matching coefficients read

$$
C_{Q_{i} \tilde{Q}_{j}}^{(0)}=\delta_{i j}
$$

\footnotetext{
${ }^{4}$ Note that [89] contains a misprint that has been identified in [76].
} 
at $\mathrm{LO}$ and

$$
C_{Q_{i} \tilde{Q}_{j}}^{(1)}=\left(\begin{array}{cccc}
-4 L_{\mu}-\frac{32}{3} & \frac{16}{3} & -\frac{a_{1}}{4}-3 L_{\mu}-13 & -2 \\
0 & 4 L_{\mu}+\frac{16}{3} & -\frac{3}{2} & -\frac{a_{2}}{4}+3 L_{\mu}-1 \\
-\frac{a_{1}}{18}-\frac{2 L_{\mu}}{3}-\frac{26}{9} & -\frac{4}{9} & -\frac{7 a_{1}}{24}+\frac{3 L_{\mu}}{2}+\frac{7}{6} & -3 \\
-\frac{1}{3} & -\frac{a_{2}}{18}+\frac{2 L_{\mu}}{3}-\frac{2}{9} & -\frac{1}{4} & -\frac{7 a_{2}}{24}-\frac{3 L_{\mu}}{2}-\frac{29}{6}
\end{array}\right),
$$

at NLO where we have set $N_{c}=3$ for brevity.

\section{B Inputs and detailed overview of uncertainties}

\begin{tabular}{|c|cc|}
\hline Parameter & Value & Source \\
\hline $\bar{m}_{b}\left(\bar{m}_{b}\right)$ & $\left(4.203_{-0.034}^{+0.016}\right) \mathrm{GeV}$ & {$[61,62]$} \\
$m_{b}^{\mathrm{PS}}(2 \mathrm{GeV})$ & $\left(4.532_{-0.039}^{+0.013}\right) \mathrm{GeV}$ & {$[61,62]$} \\
$m_{b}^{1 \mathrm{~S}}$ & $\left(4.66_{-0.03}^{+0.04}\right) \mathrm{GeV}$ & {$[60]$} \\
$m_{b}^{\mathrm{kin}}(1 \mathrm{GeV})$ & $(4.553 \pm 0.020) \mathrm{GeV}$ & {$[90]$} \\
$\bar{m}_{c}\left(\bar{m}_{c}\right)$ & $(1.279 \pm 0.013) \mathrm{GeV}$ & {$[91]$} \\
$\alpha_{s}\left(M_{Z}\right)$ & $0.1181 \pm 0.0011$ & {$[60]$} \\
$V_{u s}$ & $0.2248 \pm 0.0006$ & {$[60]$} \\
$V_{u b}$ & $0.00409 \pm 0.00039$ & {$[60]$} \\
$V_{c b}$ & $0.0405 \pm 0.0015$ & {$[60]$} \\
$\gamma$ & $\left(73.2_{-7.0}^{+6.3}\right)^{\circ}$ & {$[60]$} \\
$f_{B}$ & $(189 \pm 4) \mathrm{MeV}$ & {$[60]^{5}$} \\
$f_{B_{s}}$ & $(227.2 \pm 3.4) \mathrm{MeV}$ & {$[60]$} \\
$f_{D}$ & $(203.7 \pm 4.8) \mathrm{MeV}$ & {$[60]^{6}$} \\
\hline
\end{tabular}

Table 1. Input values for parameters.

\begin{tabular}{|l|ccccccc|}
\hline$\Delta B=2$ & $\bar{\Lambda}$ & intrinsic SR & condensates & $\mu_{\rho}$ & $1 / m_{b}$ & $\mu_{m}$ & $a_{i}$ \\
\hline $\bar{B}_{Q_{1}}$ & ${ }_{-0.002}^{+0.001}$ & \pm 0.018 & \pm 0.004 & ${ }_{-0.022}^{+0.011}$ & \pm 0.010 & ${ }_{-0.039}^{+0.045}$ & ${ }_{-0.007}^{+0.007}$ \\
$\bar{B}_{Q_{2}}$ & ${ }_{-0.017}^{+0.014}$ & $\mp 0.020$ & \pm 0.004 & ${ }_{-0.019}^{+0.012}$ & \pm 0.010 & ${ }_{-0.062}^{+0.071}$ & ${ }_{-0.015}^{+0.015}$ \\
$\bar{B}_{Q_{3}}$ & ${ }_{-0.074}^{+0.060}$ & \pm 0.107 & \pm 0.023 & ${ }_{-0.008}^{+0.016}$ & \pm 0.010 & ${ }_{-0.069}^{+0.006}$ & ${ }_{-0.052}^{+0.053}$ \\
$\bar{B}_{Q_{4}}$ & ${ }_{-0.006}^{+0.007}$ & \pm 0.021 & \pm 0.011 & ${ }_{-0.003}^{+0.003}$ & \pm 0.010 & ${ }_{-0.079}^{+0.088}$ & ${ }_{-0.006}^{+0.005}$ \\
$\bar{B}_{Q_{5}}$ & ${ }_{-0.015}^{+0.019}$ & \pm 0.018 & \pm 0.009 & ${ }_{-0.006}^{+0.004}$ & \pm 0.010 & ${ }_{-0.068}^{+0.077}$ & ${ }_{-0.012}^{+0.012}$ \\
\hline
\end{tabular}

Table 2. Individual errors for the Bag parameters of the $\Delta B=2$ matrix elements.

\footnotetext{
${ }^{5}$ We take the mean of $f_{B^{+}}$and $f_{B^{0}}$.

${ }^{6}$ We use the 'experimental' value instead of the lattice average, since the former is in significantly better agreement with sum rule results [79-82].
} 


\begin{tabular}{|l|ccccccc|}
\hline$\Delta B=0$ & $\bar{\Lambda}$ & intrinsic SR & condensates & $\mu_{\rho}$ & $1 / m_{b}$ & $\mu_{m}$ & $a_{i}$ \\
\hline $\bar{B}_{1}$ & ${ }_{-0.002}^{+0.003}$ & \pm 0.019 & \pm 0.002 & ${ }_{-0.002}^{+0.002}$ & \pm 0.010 & ${ }_{-0.052}^{+0.060}$ & ${ }_{-0.003}^{+0.002}$ \\
$\bar{B}_{2}$ & ${ }_{-0.001}^{+0.001}$ & $\mp 0.020$ & \pm 0.002 & ${ }_{-0.001}^{+0.000}$ & \pm 0.010 & ${ }_{-0.076}^{+0.084}$ & ${ }_{-0.002}^{+0.001}$ \\
$\bar{\epsilon}_{1}$ & ${ }_{-0.007}^{+0.006}$ & \pm 0.022 & \pm 0.003 & ${ }_{-0.003}^{+0.003}$ & \pm 0.010 & ${ }_{-0.012}^{+0.010}$ & ${ }_{-0.007}^{+0.006}$ \\
$\bar{\epsilon}_{2}$ & ${ }_{-0.006}^{+0.005}$ & \pm 0.017 & \pm 0.003 & ${ }_{-0.001}^{+0.002}$ & \pm 0.010 & ${ }_{-0.002}^{+0.001}$ & ${ }_{-0.004}^{+0.003}$ \\
\hline
\end{tabular}

Table 3. Individual errors for the Bag parameters of the $\Delta B=0$ matrix elements.

\begin{tabular}{|l|ccccccc|}
\hline$\Delta C=2$ & $\bar{\Lambda}$ & intrinsic SR & condensates & $\mu_{\rho}$ & $1 / m_{c}$ & $\mu_{m}$ & $a_{i}$ \\
\hline $\bar{B}_{Q_{1}}$ & ${ }_{-0.002}^{+0.001}$ & \pm 0.013 & \pm 0.003 & ${ }_{-0.021}^{+0.009}$ & \pm 0.030 & ${ }_{-0.021}^{+0.039}$ & \pm 0.003 \\
$\bar{B}_{Q_{2}}$ & ${ }_{-0.014}^{+0.011}$ & $\mp 0.015$ & \pm 0.003 & ${ }_{-0.016}^{+0.010}$ & \pm 0.030 & ${ }_{-0.050}^{+0.092}$ & \pm 0.012 \\
$\bar{B}_{Q_{3}}$ & ${ }_{-0.045}^{+0.037}$ & \pm 0.059 & \pm 0.013 & ${ }_{-0.016}^{+0.016}$ & \pm 0.030 & ${ }_{-0.059}^{+0.116}$ & \pm 0.016 \\
$\bar{B}_{Q_{4}}$ & ${ }_{-0.005}^{+0.006}$ & \pm 0.017 & \pm 0.009 & ${ }_{-0.003}^{+0.003}$ & \pm 0.030 & ${ }_{-0.071}^{+0.131}$ & \pm 0.004 \\
$\bar{B}_{Q_{5}}$ & ${ }_{-0.012}^{+0.014}$ & \pm 0.014 & \pm 0.007 & ${ }_{-0.005}^{+0.004}$ & \pm 0.030 & ${ }_{-0.069}^{+0.127}$ & \pm 0.004 \\
\hline
\end{tabular}

Table 4. Individual errors for the Bag parameters of the $\Delta C=2$ matrix elements.

\begin{tabular}{|l|ccccccc|}
\hline$\Delta C=0$ & $\bar{\Lambda}$ & intrinsic SR & condensates & $\mu_{\rho}$ & $1 / m_{c}$ & $\mu_{m}$ & $a_{i}$ \\
\hline $\bar{B}_{1}$ & ${ }_{-0.003}^{+0.004}$ & \pm 0.017 & \pm 0.002 & ${ }_{-0.002}^{+0.002}$ & \pm 0.030 & ${ }_{-0.037}^{+0.068}$ & ${ }_{-0.005}^{+0.003}$ \\
$\bar{B}_{2}$ & ${ }_{-0.000}^{+0.001}$ & $\mp 0.015$ & \pm 0.001 & ${ }_{-0.000}^{+0.000}$ & \pm 0.030 & ${ }_{-0.065}^{+0.120}$ & ${ }_{-0.001}^{+0.000}$ \\
$\bar{\epsilon}_{1}$ & ${ }_{-0.008}^{+0.007}$ & \pm 0.024 & \pm 0.004 & ${ }_{-0.004}^{+0.003}$ & \pm 0.030 & ${ }_{-0.022}^{+0.012}$ & ${ }_{-0.000}^{+0.006}$ \\
$\bar{\epsilon}_{2}$ & ${ }_{-0.004}^{+0.003}$ & \pm 0.011 & \pm 0.002 & ${ }_{-0.001}^{+0.001}$ & \pm 0.030 & ${ }_{-0.000}^{+0.000}$ & ${ }_{-0.002}^{+0.0001}$ \\
\hline
\end{tabular}

Table 5. Individual errors for the Bag parameters of the $\Delta C=0$ matrix elements. 


\begin{tabular}{|l|ccc|}
\hline & $\Delta M_{s}^{\mathrm{SM}}\left[\mathrm{ps}^{-1}\right]$ & $\Delta \Gamma_{s}^{\mathrm{PS}}\left[\mathrm{ps}^{-1}\right]$ & $a_{\mathrm{sl}}^{s, \mathrm{PS}}\left[10^{-5}\right]$ \\
\hline $\bar{B}_{Q_{1}}$ & \pm 1.1 & \pm 0.005 & \pm 0.01 \\
$\bar{B}_{Q_{3}}$ & \pm 0.0 & \pm 0.005 & \pm 0.01 \\
$\bar{B}_{R_{0}}$ & \pm 0.0 & \pm 0.003 & \pm 0.00 \\
$\bar{B}_{R_{1}}$ & \pm 0.0 & \pm 0.000 & \pm 0.00 \\
$\bar{B}_{R_{1}^{\prime}}$ & \pm 0.0 & \pm 0.000 & \pm 0.00 \\
$\bar{B}_{R_{2}}$ & \pm 0.0 & \pm 0.016 & \pm 0.00 \\
$\bar{B}_{R_{3}}$ & \pm 0.0 & \pm 0.001 & \pm 0.02 \\
$\bar{B}_{R_{3}^{\prime}}$ & \pm 0.0 & \pm 0.000 & \pm 0.05 \\
$f_{B_{s}}$ & \pm 0.5 & \pm 0.002 & \pm 0.00 \\
$\mu_{1}$ & \pm 0.0 & ${ }_{-0.018}^{+0.007}$ & ${ }_{-0.08}^{+0.04}$ \\
$\mu_{2}$ & \pm 0.1 & ${ }_{-0.002}^{+0.000}$ & \pm 0.01 \\
$m_{b}$ & \pm 0.0 & ${ }_{-0.000}^{+0.000}$ & \pm 0.01 \\
$m_{c}$ & \pm 0.0 & ${ }_{-0.000}^{+0.000}$ & \pm 0.06 \\
$\alpha_{s}$ & \pm 0.0 & \pm 0.000 & \pm 0.04 \\
$\mathrm{CKM}$ & ${ }_{-1.3}^{+1.4}$ & \pm 0.006 & ${ }_{-0.22}^{+0.21}$ \\
\hline
\end{tabular}

Table 6. Individual errors for the $B_{s}$ mixing observables. 


\begin{tabular}{|l|ccc|}
\hline & $\Delta M_{d}^{\mathrm{SM}}\left[\mathrm{ps}^{-1}\right]$ & $\Delta \Gamma_{d}^{\mathrm{PS}}\left[10^{-3} \mathrm{ps}^{-1}\right]$ & $a_{\mathrm{sl}}^{d, \mathrm{PS}}\left[10^{-4}\right]$ \\
\hline $\bar{B}_{Q_{1}}$ & ${ }_{-0.03}^{+0.04}$ & \pm 0.16 & \pm 0.02 \\
$\bar{B}_{Q_{3}}$ & \pm 0.00 & +0.17 & \pm 0.03 \\
$\bar{B}_{R_{0}}$ & \pm 0.00 & \pm 0.11 & \pm 0.01 \\
$\bar{B}_{R_{1}}$ & \pm 0.00 & \pm 0.01 & \pm 0.00 \\
$\bar{B}_{R_{1}^{\prime}}$ & \pm 0.00 & \pm 0.01 & \pm 0.00 \\
$\bar{B}_{R_{2}}$ & \pm 0.00 & \pm 0.54 & \pm 0.00 \\
$\bar{B}_{R_{3}}$ & \pm 0.00 & \pm 0.00 & \pm 0.04 \\
$\bar{B}_{R_{3}^{\prime}}$ & \pm 0.00 & \pm 0.01 & \pm 0.09 \\
$f_{B}$ & \pm 0.03 & \pm 0.11 & \pm 0.00 \\
$\mu_{1}$ & \pm 0.00 & ${ }_{-0.62}^{+0.24}$ & ${ }_{-0.07}^{+0.17}$ \\
$\mu_{2}$ & \pm 0.00 & ${ }_{-0.08}^{+0.00}$ & ${ }_{-0.03}^{+0.01}$ \\
$m_{b}$ & \pm 0.00 & ${ }_{-0.03}^{+0.01}$ & ${ }_{-0.03}^{+0.01}$ \\
$m_{c}$ & \pm 0.00 & ${ }_{-0.02}^{+0.01}$ & \pm 0.13 \\
$\alpha_{s}$ & \pm 0.00 & ${ }_{-0.01}$ & \pm 0.08 \\
$\mathrm{CKM}$ & \pm 0.08 & ${ }_{-0.37}^{+0.38}$ & ${ }_{-0.44}^{+0.47}$ \\
\hline
\end{tabular}

Table 7. Individual errors for the $B_{d}$ mixing observables.

\begin{tabular}{|cccccccc|}
\hline $\bar{B}_{1}$ & $\bar{B}_{2}$ & $\bar{\epsilon}_{1}$ & $\bar{\epsilon}_{2}$ & $\rho_{3}$ & $\rho_{4}$ & $\sigma_{3}$ & $\sigma_{4}$ \\
\hline \pm 0.002 & \pm 0.000 & ${ }_{-0.015}^{+0.016}$ & \pm 0.004 & \pm 0.001 & \pm 0.000 & \pm 0.013 & \pm 0.000 \\
\hline \hline$f_{B}$ & $\mu_{1}$ & $\mu_{0}$ & $m_{b}$ & $m_{c}$ & $\alpha_{s}$ & CKM & \\
\hline${ }_{-0.003}^{+0.004}$ & ${ }_{-0.013}^{+0.000}$ & ${ }_{-0.006}^{+0.000}$ & ${ }_{-0.001}^{+0.000}$ & \pm 0.000 & \pm 0.002 & \pm 0.006 & \\
\hline
\end{tabular}

Table 8. Individual errors for the ratio $\tau\left(B^{+}\right) / \tau\left(B^{0}\right)$ in the PS mass scheme.

\begin{tabular}{|cccccccc|}
\hline $\bar{B}_{1}$ & $\bar{B}_{2}$ & $\bar{\epsilon}_{1}$ & $\bar{\epsilon}_{2}$ & $\rho_{3}$ & $\rho_{4}$ & $\sigma_{3}$ & $\sigma_{4}$ \\
\hline${ }_{-0.05}^{+0.07}$ & \pm 0.00 & ${ }_{-0.47}^{+0.52}$ & \pm 0.017 & \pm 0.05 & \pm 0.00 & \pm 0.46 & \pm 0.00 \\
\hline \hline$f_{B}$ & $\mu_{1}$ & $\mu_{0}$ & $m_{c}$ & $m_{s}$ & $\alpha_{s}$ & $\mathrm{CKM}$ & \\
\hline \pm 0.08 & ${ }_{-0.40}^{+0.07}$ & ${ }_{-0.21}^{+0.08}$ & \pm 0.08 & \pm 0.00 & ${ }_{0.06}^{+0.07}$ & \pm 0.00 & \\
\hline
\end{tabular}

Table 9. Individual errors for the ratio $\tau\left(D^{+}\right) / \tau\left(D^{0}\right)$ in the PS mass scheme. 
Open Access. This article is distributed under the terms of the Creative Commons Attribution License (CC-BY 4.0), which permits any use, distribution and reproduction in any medium, provided the original author(s) and source are credited.

\section{References}

[1] M. Artuso, G. Borissov and A. Lenz, CP violation in the $B_{s}^{0}$ system, Rev. Mod. Phys. 88 (2016) 045002 [arXiv: 1511.09466] [INSPIRE].

[2] H.M. Asatrian, A. Hovhannisyan, U. Nierste and A. Yeghiazaryan, Towards next-to-next-to-leading-log accuracy for the width difference in the $B_{s}-\bar{B}_{s}$ system: fermionic contributions to order $\left(m_{c} / m_{b}\right)^{0}$ and $\left(m_{c} / m_{b}\right)^{1}$, JHEP 10 (2017) 191 [arXiv:1709.02160] [INSPIRE].

[3] E. Dalgic et al., $B_{s}^{0}-\bar{B}_{s}^{0}$ mixing parameters from unquenched lattice $Q C D$, Phys. Rev. D 76 (2007) 011501 [hep-lat/0610104] [INSPIRE].

[4] ETM collaboration, N. Carrasco et al., B-physics from $N_{f}=2$ tmQCD: the Standard Model and beyond, JHEP 03 (2014) 016 [arXiv: 1308.1851] [INSPIRE].

[5] Fermilab Lattice and MiLC collaborations, A. Bazavov et al., $B_{(s)}^{0}$-mixing matrix elements from lattice QCD for the Standard Model and beyond, Phys. Rev. D 93 (2016) 113016 [arXiv: 1602.03560] [INSPIRE].

[6] T. Jubb, M. Kirk, A. Lenz and G. Tetlalmatzi-Xolocotzi, On the ultimate precision of meson mixing observables, Nucl. Phys. B 915 (2017) 431 [arXiv:1603.07770] [INSPIRE].

[7] M.A. Shifman, A.I. Vainshtein and V.I. Zakharov, QCD and Resonance Physics. Theoretical Foundations, Nucl. Phys. B 147 (1979) 385 [InSPIRE].

[8] M.A. Shifman, A.I. Vainshtein and V.I. Zakharov, QCD and Resonance Physics: Applications, Nucl. Phys. B 147 (1979) 448 [inSPIRE].

[9] K.G. Chetyrkin, A.L. Kataev, A.B. Krasulin and A.A. Pivovarov, Calculation of the $K^{0}-\bar{K}^{0}$ mixing parameter via the $Q C D$ sum rules at finite energies, Phys. Lett. B 174 (1986) 104 [hep-ph/0103230] [INSPIRE].

[10] J.G. Körner, A.I. Onishchenko, A.A. Petrov and A.A. Pivovarov, $B^{0}-\bar{B}^{0}$ mixing beyond factorization, Phys. Rev. Lett. 91 (2003) 192002 [hep-ph/0306032] [INSPIRE].

[11] A.G. Grozin, R. Klein, T. Mannel and A.A. Pivovarov, $B^{0}-\bar{B}^{0}$ mixing at next-to-leading order, Phys. Rev. D 94 (2016) 034024 [arXiv: 1606.06054] [INSPIRE].

[12] T. Mannel, B.D. Pecjak and A.A. Pivovarov, Analyzing $B_{s}-\bar{B}_{s}$ mixing: Non-perturbative contributions to bag parameters from sum rules, [hep-ph/0703244].

[13] T. Mannel, B.D. Pecjak and A.A. Pivovarov, Sum rule estimate of the subleading non-perturbative contributions to $B_{s}-\bar{B}_{s}$ mixing, Eur. Phys. J. C 71 (2011) 1607 [INSPIRE].

[14] M.S. Baek, J. Lee, C. Liu and H.S. Song, Four quark operators relevant to B meson lifetimes from QCD sum rules, Phys. Rev. D 57 (1998) 4091 [hep-ph/9709386] [INSPIRE].

[15] H.-Y. Cheng and K.-C. Yang, Nonspectator effects and B meson lifetimes from a field theoretic calculation, Phys. Rev. D 59 (1999) 014011 [hep-ph/9805222] [INSPIRE].

[16] A. Lenz, Lifetimes and heavy quark expansion, Int. J. Mod. Phys. A 30 (2015) 1543005 [arXiv: 1405.3601] [INSPIRE]. 
[17] V.A. Khoze and M.A. Shifman, Heavy Quarks, Sov. Phys. Usp. 26 (1983) 387 [InSPIRE].

[18] M.A. Shifman and M.B. Voloshin, Preasymptotic Effects in Inclusive Weak Decays of Charmed Particles, Sov. J. Nucl. Phys. 41 (1985) 120 [Yad. Fiz. 41 (1985) 187] [InSPIRE].

[19] I.I.Y. Bigi and N.G. Uraltsev, Gluonic enhancements in non-spectator beauty decays: An inclusive mirage though an exclusive possibility, Phys. Lett. B 280 (1992) 271 [INSPIRE].

[20] I.I.Y. Bigi, N.G. Uraltsev and A.I. Vainshtein, Nonperturbative corrections to inclusive beauty and charm decays: QCD versus phenomenological models, Phys. Lett. B 293 (1992) 430 [Erratum ibid. B 297 (1992) 477] [hep-ph/9207214] [INSPIRE].

[21] F. Krinner, A. Lenz and T. Rauh, The inclusive decay $b \rightarrow c \bar{c} s$ revisited, Nucl. Phys. B 876 (2013) 31 [arXiv: 1305.5390] [INSPIRE].

[22] UKQCD collaboration, M. Di Pierro and C.T. Sachrajda, A Lattice study of spectator effects in inclusive decays of B mesons, Nucl. Phys. B 534 (1998) 373 [hep-lat/9805028] [INSPIRE].

[23] D. Becirevic, Theoretical progress in describing the B meson lifetimes, PoS(HEP2001) 098 [hep-ph/0110124] [INSPIRE].

[24] M. Bobrowski, A. Lenz, J. Riedl and J. Rohrwild, How Large Can the SM Contribution to CP-violation in $D^{0}-\bar{D}^{0}$ Mixing Be?, JHEP 03 (2010) 009 [arXiv: 1002.4794] [INSPIRE].

[25] I.I.Y. Bigi and N.G. Uraltsev, $D^{0}-\bar{D}^{0}$ oscillations as a probe of quark hadron duality, Nucl. Phys. B 592 (2001) 92 [hep-ph/0005089] [INSPIRE].

[26] H. Georgi, D- $\bar{D}$ mixing in heavy quark effective field theory, Phys. Lett. B 297 (1992) 353 [hep-ph/9209291] [INSPIRE].

[27] T. Ohl, G. Ricciardi and E.H. Simmons, $D-\bar{D}$ mixing in heavy quark effective field theory: The sequel, Nucl. Phys. B 403 (1993) 605 [hep-ph/9301212] [InSPIRE].

[28] M. Bobrowski, A. Lenz and T. Rauh, Short distance D-Dbar mixing, arXiv:1208.6438 [INSPIRE].

[29] A. Lenz and T. Rauh, D-meson lifetimes within the heavy quark expansion, Phys. Rev. D 88 (2013) 034004 [arXiv: 1305.3588] [inSPIRE].

[30] N. Carrasco et al., $D^{0}-\bar{D}^{0}$ mixing in the standard model and beyond from $N_{f}=2$ twisted mass QCD, Phys. Rev. D 90 (2014) 014502 [arXiv: 1403.7302] [INSPIRE].

[31] ETM collaboration, N. Carrasco et al., $\Delta S=2$ and $\Delta C=2$ bag parameters in the standard model and beyond from $N_{f}=2+1+1$ twisted-mass lattice QCD, Phys. Rev. D 92 (2015) 034516 [arXiv: 1505.06639] [INSPIRE].

[32] A. Bazavov et al., Short-distance matrix elements for $D^{0}$-meson mixing for $N_{f}=2+1$ lattice QCD, arXiv:1706.04622 [INSPIRE].

[33] J.M. Flynn, O.F. Hernandez and B.R. Hill, Renormalization of four fermion operators determining B $\bar{B}$ mixing on the lattice, Phys. Rev. D 43 (1991) 3709 [inSPIRE].

[34] G. Buchalla, Renormalization of $\Delta B=2$ transitions in the static limit beyond leading logarithms, Phys. Lett. B 395 (1997) 364 [hep-ph/9608232] [INSPIRE].

[35] M. Ciuchini, E. Franco and V. Giménez, Next-to-leading order renormalization of the $\Delta B=2$ operators in the static theory, Phys. Lett. B 388 (1996) 167 [hep-ph/9608204] [INSPIRE].

[36] J.C. Collins, Renormalization: An Introduction to Renormalization, The Renormalization Group, and the Operator Product Expansion, Cambridge University Press, (1984). 
[37] A.J. Buras and P.H. Weisz, QCD Nonleading Corrections to Weak Decays in Dimensional Regularization and 't Hooft-Veltman Schemes, Nucl. Phys. B 333 (1990) 66 [InSPIRE].

[38] S. Herrlich and U. Nierste, Evanescent operators, scheme dependences and double insertions, Nucl. Phys. B 455 (1995) 39 [hep-ph/9412375] [INSPIRE].

[39] M. Beneke, G. Buchalla, C. Greub, A. Lenz and U. Nierste, Next-to-leading order QCD corrections to the lifetime difference of $B_{s}$ mesons, Phys. Lett. B 459 (1999) 631 [hep-ph/9808385] [INSPIRE].

[40] M. Beneke, G. Buchalla and I. Dunietz, Width Difference in the $B_{s}-\bar{B}_{s}$ System, Phys. Rev. D 54 (1996) 4419 [Erratum ibid. D 83 (2011) 119902] [hep-ph/9605259] [INSPIRE].

[41] P. Nogueira, Automatic Feynman graph generation, J. Comput. Phys. 105 (1993) 279 [INSPIRE].

[42] M. Jamin and M.E. Lautenbacher, TRACER: Version 1.1: A Mathematica package for gamma algebra in arbitrary dimensions, Comput. Phys. Commun. 74 (1993) 265 [INSPIRE].

[43] H.H. Patel, Package-X: A Mathematica package for the analytic calculation of one-loop integrals, Comput. Phys. Commun. 197 (2015) 276 [arXiv:1503.01469] [INSPIRE].

[44] H.H. Patel, Package-X 2.0: A Mathematica package for the analytic calculation of one-loop integrals, Comput. Phys. Commun. 218 (2017) 66 [arXiv:1612.00009] [INSPIRE].

[45] F. Gabbiani, E. Gabrielli, A. Masiero and L. Silvestrini, A complete analysis of FCNC and CP constraints in general SUSY extensions of the standard model, Nucl. Phys. B 477 (1996) 321 [hep-ph/9604387] [INSPIRE].

[46] A. Lenz and U. Nierste, Theoretical update of $B_{s}-\bar{B}_{s}$ mixing, JHEP 06 (2007) 072 [hep-ph/0612167] [INSPIRE].

[47] E. Eichten and B.R. Hill, An Effective Field Theory for the Calculation of Matrix Elements Involving Heavy Quarks, Phys. Lett. B 234 (1990) 511 [INSPIRE].

[48] A.V. Smirnov, Algorithm FIRE - Feynman Integral REduction, JHEP 10 (2008) 107 [arXiv: 0807.3243] [INSPIRE].

[49] A.V. Smirnov and V.A. Smirnov, FIRE4, LiteRed and accompanying tools to solve integration by parts relations, Comput. Phys. Commun. 184 (2013) 2820 [arXiv:1302.5885] [INSPIRE].

[50] A.V. Smirnov, FIRE5: a C++ implementation of Feynman Integral REduction, Comput. Phys. Commun. 189 (2015) 182 [arXiv:1408.2372] [INSPIRE].

[51] K.G. Chetyrkin and F.V. Tkachov, Integration by Parts: The Algorithm to Calculate $\beta$-functions in 4 Loops, Nucl. Phys. B 192 (1981) 159 [InSPIRE].

[52] S. Laporta, High precision calculation of multiloop Feynman integrals by difference equations, Int. J. Mod. Phys. A 15 (2000) 5087 [hep-ph/0102033] [inSPIRE].

[53] A.G. Grozin and R.N. Lee, Three-loop HQET vertex diagrams for $B^{0}-\bar{B}^{0}$ mixing, JHEP 02 (2009) 047 [arXiv: 0812.4522] [inSPIRE].

[54] P. Ball and V.M. Braun, Next-to-leading order corrections to meson masses in the heavy quark effective theory, Phys. Rev. D 49 (1994) 2472 [hep-ph/9307291] [INSPIRE].

[55] D.J. Broadhurst and A.G. Grozin, Operator product expansion in static quark effective field theory: Large perturbative correction, Phys. Lett. B 274 (1992) 421 [hep-ph/9908363] [INSPIRE]. 
[56] E. Bagan, P. Ball, V.M. Braun and H.G. Dosch, QCD sum rules in the effective heavy quark theory, Phys. Lett. B 278 (1992) 457 [inSPIRE].

[57] M. Neubert, Heavy meson form-factors from QCD sum rules, Phys. Rev. D 45 (1992) 2451 [INSPIRE].

[58] K.G. Chetyrkin, J.H. Kühn and M. Steinhauser, RunDec: A Mathematica package for running and decoupling of the strong coupling and quark masses, Comput. Phys. Commun. 133 (2000) 43 [hep-ph/0004189] [INSPIRE].

[59] F. Herren and M. Steinhauser, Version 3 of RunDec and CRunDec, arXiv:1703.03751 [INSPIRE].

[60] Particle Data Group collaboration, C. Patrignani et al., Review of Particle Physics, Chin. Phys. C 40 (2016) 100001 [inSPIRE].

[61] M. Beneke, A. Maier, J. Piclum and T. Rauh, The bottom-quark mass from non-relativistic sum rules at NNNLO, Nucl. Phys. B 891 (2015) 42 [arXiv:1411.3132] [INSPIRE].

[62] M. Beneke, A. Maier, J. Piclum and T. Rauh, NNNLO determination of the bottom-quark mass from non-relativistic sum rules, PoS (RADCOR2015) 035 [arXiv: 1601.02949] [INSPIRE].

[63] P.A. Baikov, K.G. Chetyrkin and J.H. Kühn, Five-Loop Running of the QCD coupling constant, Phys. Rev. Lett. 118 (2017) 082002 [arXiv: 1606.08659] [INSPIRE].

[64] F. Herzog, B. Ruijl, T. Ueda, J.A.M. Vermaseren and A. Vogt, The five-loop $\beta$-function of Yang-Mills theory with fermions, JHEP 02 (2017) 090 [arXiv:1701.01404] [INSPIRE].

[65] T. Luthe, A. Maier, P. Marquard and Y. Schröder, Complete renormalization of QCD at five loops, JHEP 03 (2017) 020 [arXiv: 1701.07068] [INSPIRE].

[66] T. Luthe, A. Maier, P. Marquard and Y. Schröder, The five-loop $\beta$-function for a general gauge group and anomalous dimensions beyond Feynman gauge, JHEP 10 (2017) 166 [arXiv: 1709.07718] [INSPIRE].

[67] K.G. Chetyrkin, G. Falcioni, F. Herzog and J.A.M. Vermaseren, Five-loop renormalisation of QCD in covariant gauges, JHEP 10 (2017) 179 [arXiv:1709.08541] [INSPIRE].

[68] S. Aoki et al., Review of lattice results concerning low-energy particle physics, Eur. Phys. J. C 77 (2017) 112 [arXiv: 1607.00299] [INSPIRE].

[69] Y. Amhis et al., Averages of b-hadron, c-hadron and $\tau$-lepton properties as of summer 2016, arXiv: 1612.07233 [INSPIRE].

[70] A.G. Grozin, T. Mannel and A.A. Pivovarov, Towards a Next-to-Next-to-Leading Order analysis of matching in $B^{0}-\bar{B}^{0}$ mixing, Phys. Rev. D 96 (2017) 074032 [arXiv: 1706. 05910] [INSPIRE].

[71] M. Beneke, A quark mass definition adequate for threshold problems, Phys. Lett. B 434 (1998) 115 [hep-ph/9804241] [INSPIRE].

[72] A.H. Hoang, Z. Ligeti and A.V. Manohar, B decay and the Upsilon mass, Phys. Rev. Lett. 82 (1999) 277 [hep-ph/9809423] [inSPIRE].

[73] I.I.Y. Bigi, M.A. Shifman, N. Uraltsev and A.I. Vainshtein, High power $n$ of $m_{b}$ in beauty widths and $N=5 \rightarrow \infty$ limit, Phys. Rev. D 56 (1997) 4017 [hep-ph/9704245] [INSPIRE].

[74] S. Laplace, Z. Ligeti, Y. Nir and G. Perez, Implications of the CP asymmetry in semileptonic B decay, Phys. Rev. D 65 (2002) 094040 [hep-ph/0202010] [INSPIRE]. 
[75] M. Beneke, G. Buchalla, C. Greub, A. Lenz and U. Nierste, The $B^{+}-B_{d}^{0}$ lifetime difference beyond leading logarithms, Nucl. Phys. B 639 (2002) 389 [hep-ph/0202106] [InSPIRE].

[76] M. Ciuchini, E. Franco, V. Lubicz and F. Mescia, Next-to-leading order QCD corrections to spectator effects in lifetimes of beauty hadrons, Nucl. Phys. B 625 (2002) 211 [hep-ph/0110375] [INSPIRE].

[77] E. Franco, V. Lubicz, F. Mescia and C. Tarantino, Lifetime ratios of beauty hadrons at the next-to-leading order in QCD, Nucl. Phys. B 633 (2002) 212 [hep-ph/0203089] [INSPIRE].

[78] F. Gabbiani, A.I. Onishchenko and A.A. Petrov, Spectator effects and lifetimes of heavy hadrons, Phys. Rev. D 70 (2004) 094031 [hep-ph/0407004] [INSPIRE].

[79] Z.-G. Wang, Analysis of the masses and decay constants of the heavy-light mesons with QCD sum rules, Eur. Phys. J. C 75 (2015) 427 [arXiv: 1506.01993] [INSPIRE].

[80] P. Gelhausen, A. Khodjamirian, A.A. Pivovarov and D. Rosenthal, Decay constants of heavy-light vector mesons from QCD sum rules, Phys. Rev. D 88 (2013) 014015 [Erratum ibid. D 89 (2014) 099901] [Erratum ibid. D 91 (2015) 099901] [arXiv: 1305.5432] [INSPIRE].

[81] S. Narison, A fresh look into $m_{c, b}$ and precise $f_{D_{(s)}, B_{(s)}}$ from heavy-light $Q C D$ spectral sum rules, Phys. Lett. B 718 (2013) 1321 [arXiv:1209.2023] [INSPIRE].

[82] W. Lucha, D. Melikhov and S. Simula, OPE, charm-quark mass and decay constants of $D$ and $D_{s}$ mesons from QCD sum rules, Phys. Lett. B 701 (2011) 82 [arXiv:1101.5986] [INSPIRE].

[83] M. Beneke, Y. Kiyo, A. Maier and J. Piclum, Near-threshold production of heavy quarks with QQbar_threshold, Comput. Phys. Commun. 209 (2016) 96 [arXiv:1605.03010] [INSPIRE].

[84] M. Beneke, A. Maier, T. Rauh and P. Ruiz-Femenia, Non-resonant and electroweak NNLO correction to the $e^{+} e^{-}$top anti-top threshold, arXiv:1711.10429 [INSPIRE].

[85] E. Gamiz, J. Shigemitsu and H. Trottier, Four Fermion Operator Matching with NRQCD Heavy and AsqTad Light Quarks, Phys. Rev. D 77 (2008) 114505 [arXiv:0804.1557] [INSPIRE].

[86] C. Monahan, E. Gamiz, R. Horgan and J. Shigemitsu, Matching lattice and continuum four-fermion operators with nonrelativistic QCD and highly improved staggered quarks, Phys. Rev. D 90 (2014) 054015 [arXiv: 1407.4040] [INSPIRE].

[87] M. Ciuchini, E. Franco, V. Lubicz, G. Martinelli, I. Scimemi and L. Silvestrini, Next-to-leading order QCD corrections to $\Delta F=2$ effective Hamiltonians, Nucl. Phys. B 523 (1998) 501 [hep-ph/9711402] [INSPIRE].

[88] A.J. Buras, M. Misiak and J. Urban, Two loop QCD anomalous dimensions of flavor changing four quark operators within and beyond the standard model, Nucl. Phys. B 586 (2000) 397 [hep-ph/0005183] [INSPIRE].

[89] M. Neubert and C.T. Sachrajda, Spectator effects in inclusive decays of beauty hadrons, Nucl. Phys. B 483 (1997) 339 [hep-ph/9603202] [INSPIRE].

[90] A. Alberti, P. Gambino, K.J. Healey and S. Nandi, Precision Determination of the Cabibbo-Kobayashi-Maskawa Element $V_{c b}$, Phys. Rev. Lett. 114 (2015) 061802 [arXiv: 1411.6560] [INSPIRE].

[91] K.G. Chetyrkin et al., Charm and Bottom Quark Masses: An Update, Phys. Rev. D 80 (2009) 074010 [arXiv:0907.2110] [INSPIRE]. 